\title{
Glitches in Anomalous X-ray Pulsars
}

\author{
Rim Dib ${ }^{1}$, Victoria M. Kaspi ${ }^{1}$, and Fotis P. Gavriil ${ }^{2}$
}

\begin{abstract}
We report on 8.7 and 7.6 yr of Rossi X-ray Timing Explorer (RXTE) observations of the Anomalous X-ray Pulsars (AXPs) RXS J170849.0-400910 and 1E 1841-045, respectively. These observations, part of a larger RXTE AXP monitoring program, have allowed us to study the long-term timing, pulsed flux, and pulse profile evolution of these objects. We report on four new glitches, one from RXS J170849.0-400910 and three from 1E 1841-045. One of the glitches from 1E 1841-045 is among the largest ever seen in a neutron star in terms of fractional frequency increase. With nearly all known persistent AXPs now seen to glitch, such behavior is clearly generic to this source class. We show that in terms of fractional frequency change, AXPs are among the most actively glitching neutron stars, with glitch amplitudes in general larger than in radio pulsars. However, in terms of absolute glitch amplitude, AXP glitches are unremarkable. We show that the largest AXP glitches observed thus far have recoveries that are unusual among those of radio pulsar glitches, with the combination of recovery time scale and fraction yielding changes in spin-down rates following the glitch similar to, or larger than, the long-term average. We also observed a large longterm fractional increase in the magnitude of the spin-down rate of $1 \mathrm{E} 1841-045$, following its largest glitch, with $\Delta \dot{\nu} / \dot{\nu}=0.1$. These observations are challenging to interpret in standard glitch models, as is the frequent occurence of large glitches given AXPs' high measured temperatures. We speculate that the stellar core may be involved in the largest AXP glitches. Furthermore, we show that AXP glitches appear to fall in two classes: radiatively loud and radiatively quiet. The latter, of which the glitches of RXS J170849.0-400910 and 1E 1841-045 are examples, show little evidence for an accompanying radiative event such as a sudden flux increase or pulse profile change. We also show, however, that pulse profile and pulsed flux changes are common in these AXPs, but do not apprear closely correlated with any timing behavior.
\end{abstract}

\footnotetext{
${ }^{1}$ Department of Physics, McGill University, Montreal, QC H3A 2T8

${ }^{2}$ NASA Goddard Space Flight Center, Greenbelt, MD.
} 
Subject headings: pulsars: individual(RXS J170849.0-400910) — pulsars: individual(1E 1841-045) — stars: neutron — X-rays: stars

\section{Introduction}

The past decade has seen significant progress in our knowledge of the observational properties of Anomalous X-ray Pulsars (AXPs; see Woods \& Thompson 2006; Kaspi 2007, for recent reviews). From a timing point of view, the presence of binary companions has been practically ruled out (Mereghetti et al. 1998; Wilson et al.|1999), and subsequently their potential for great rotational stability was demonstrated (Kaspi et al. 1999), thereby allowing the discovery that AXPs can exhibit spin-up glitches (Kaspi et al. 2000; Kaspi \& Gavriil 2003; Dall'Osso et al. 2003), and large (factor of 10) torque variations (Gavriil \& Kaspi 2004). From a radiative point of view, AXPs are now known to show a variety of different variability phenomena, including long-lived flares (Gavriil \& Kaspi 2004), short SGRlike bursts (Gavriil et al. 2002, 2004; Woods et al. 2005), large outbursts (Kaspi et al. 2003; Ibrahim et al. 2004; Israel et al. 2007a; Dib et al. 2007b; Tam et al. 2006; Tam et al., submitted), and slow, low-level flux and pulse profile variability (Dib et al. 2007a; Gonzalez et al., submitted). Spectrally, though previously studied only in the soft X-ray band, AXPs are now seen in the radio band (Camilo et al. 2006), through the mid- (Wang et al. 2006) and near-IR (e.g., Israel et al. 2002; Wang \& Chakrabartv 2002; Hulleman et al. 2004; Tam et al. 2004; Rea et al. 2004; Durant \& van Kerkwijk 2005), in the optical range (e.g. Kern \& Martin 2002; Dhillon et al. 2005), up to hard X-ray energies (Kuiper et al. 2006). The evidence thus far argues strongly that AXPs, like their close cousins, the Soft Gamma Repeaters, are magnetars - young, isolated neutron stars powered by a large magnetic energy reservoir, with surface fields of $>10^{14}-10^{15} \mathrm{G}$ (Thompson \& Duncan 1996; Thompson et al. 2002).

In spite of this progress, however, many aspects of AXPs remain mysterious. Particularly so are their variability properties. What is the origin of the variety of different types of variability? Although bursts can be explained as sudden crustal yields, slower evolution (e.g., Gavriil \& Kaspi 2004; Dib et al. 2007a) has been suggested to be due to slow magnetospheric twists (Thompson et al. 2002). Some support for this picture has been argued to come from observed correlations between flux and spectral hardness (Woods et al. 2004; Rea et al. 2005; Campana et al. 2007), although Özel \& Guver (2007) argue that such a correlation need not originate uniquely from the magnetosphere and could be purely thermal. At least some radiative variability has been seen to be correlated with timing behavior. The best example of this occured in the 2002 outburst of AXP 1E 2259+586 in which the pulsar suffered a 
large spin-up glitch apparently simultaneously with a major X-ray outburst (Kaspi et al. 2003; Woods et al. 2004). Israel et al. (2007a) describe a similar radiative outburst in AXP CXOU J164710.2-455216, and report a large contemporaneous glitch, as did Dib et al. (2007b) recently for AXP 1E 1048.1-5937. By contrast, AXP RXS J170849.0-400910 exhibited two glitches with no evidence for a corresponding radiative event (Kaspi et al. 2000; Kaspi \& Gavriil 2003) although Dall'Osso et al. (2003) suggested possible low-level pulse profile changes associated with the second glitch. Campana et al. (2007) also suggested that observed flux and spectral changes may be associated with glitches and predicted a third glitch would be observed after mid-2005 on the basis of an observed flux increase and apparently correlated spectral changes.

Here we report on 8.7 and 7.6 yr of monitoring of RXS J170849.0-400910 and 1E 1841-045, respectively, using the Proportional Counter Array (PCA) aboard the Rossi X-ray Timing Explorer $(R X T E)$. We report the discovery of one new glitch and three new glitch candidates in RXS J170849.0-400910 as well as three new glitches in 1E 1841-045, including one of the largest glitches, in terms of fractional frequency increase, thus far observed in any neutron star. We also present pulsed flux time series for RXS J170849.0-400910 and 1E 1841-045 which reveal little or no evidence for correlated changes with glitches, although RXS J170849.0-400910 shows low-level pulsed flux variability at many epochs. We also report a pulse profile evolution analysis which shows that both pulsars' profiles are evolving slowly with time, though in neither case does this evolution show a clear correlation with timing behavior. These results demonstrate that AXPs RXS J170849.0-400910 and 1E 1841-045 are frequent glitchers. They also demonstrate that although AXP timing glitches can occur simultaneously with significant long-lived radiative enhancements, they need not always do so.

\section{Observations}

The results presented here were obtained using the PCA on board RXTE. The PCA consists of an array of five collimated xenon/methane multi-anode proportional counter units (PCUs) operating in the $2-60 \mathrm{keV}$ range, with a total effective area of approximately $6500 \mathrm{~cm}^{2}$ and a field of view of $\sim 1^{\circ}$ FWHM (Jahoda et al. 1996). Our 294 observations of RXS J170849.0-400910 and our 136 observations of 1E 1841-045 are of various lengths (see Tables 1 and 2). Most were obtained over a period of several years as part of a long-term monitoring program, but some are isolated observations (see Figures 1 and 2).

For the monitoring, we used the GoodXenonwithPropane data mode except during Cy-

cles 10 and 11 when we used the GoodXenon mode. Both data modes record photon arrival 
times with $1-\mu$ s resolution and bin photon energies into one of 256 channels. To maximize the signal-to-noise ratio, we analysed only those events from the top xenon layer of each PCU.

\section{Phase-Coherent Timing}

Photon arrival times at each epoch were adjusted to the solar system barycenter. Resulting arrival times were binned with 31.25-ms time resolution. In the RXS J170849.0-400910 timing analysis, we included only events in the energy range $2-6 \mathrm{keV}$, to maximize the signal-to-noise ratio of the pulse. Similarly, for 1E 1841-045 we included events in the energy range $2-11 \mathrm{keV}$.

Each barycentric binned time series was folded using an ephemeris determined iteratively by maintaining phase coherence as we describe below. Resulting pulse profiles, with 64 phase bins, were cross-correlated in the Fourier domain with a high signal-to-noise template created by adding phase-aligned profiles from all observations. The cross-correlation returned an average pulse time of arrival (TOA) for each observation corresponding to a fixed pulse phase. The pulse phase $\phi$ at any time $t$ can usually be expressed as a Taylor expansion,

$$
\phi(t)=\phi_{0}\left(t_{0}\right)+\nu_{0}\left(t-t_{0}\right)+\frac{1}{2} \dot{\nu_{0}}\left(t-t_{0}\right)^{2}+\frac{1}{6} \ddot{\nu_{0}}\left(t-t_{0}\right)^{3}+\ldots,
$$

where $\nu \equiv 1 / P$ is the pulse frequency, $\dot{\nu} \equiv d \nu / d t$, etc., and subscript " 0 " denotes a parameter evaluated at the reference epoch $t=t_{0}$. The TOAs were fitted to the above polynomial using the pulsar timing software package TEMPO1.

Note that we also searched for X-ray bursts in each 2-20 keV barycentered, binned time series using the methods described in Gavriil et al. (2004), however no bursts were found in any of our RXS J170849.0-400910 or 1E 1841-045 data sets.

\subsection{Timing Results for RXS J170849.0-400910}

Figure 3 and Table 3 summarize our results for RXS J170849.0-400910. The pulsar's spin evolution can be characterized by steady spin-down, punctuated by sudden episodes of spin-up, i.e., glitches, in addition to candidate glitch events and apparently random noise. We provide in Table 3 pulse ephemerides for inter-glitch ranges labelled as in the top panel of

\footnotetext{
${ }^{1}$ See http://www.atnf.csiro.au/research/pulsar/tempo.
} 
Figure 3. Residuals after subtraction of these models are shown in the next panel of Figure 3 . Overall the models describe the data well. However, particularly when our timing precision was highest (i.e., before 2003), some low-level but significant deviations are seen on time scales of weeks to months. Their origin is unknown but is likely related to "timing noise," commonly seen in other AXPs (e.g., Kaspi et al. 1999; Gotthelf et al. 2002) and ubiquitously in radio pulsars (e.g., Arzoumanian et al. 1994; Hobbs et al. 2004; Livingstone et al. 2005). Note that Dib et al. (2007a) performed simulations which showed that pulse profile changes similar to those observed in this source (see \$4.1) did not result in timing offsets significantly larger than our reported TOA uncertainties. Hence, the features in the timing residuals reported here are not a result of pulse profile changes.

In addition to the two previously reported glitches (which we have reanalysed, finding results consistent with those already in the literature; see Kaspi et al. 2000; Kaspi \& Gavriil 2003; Dall'Osso et al. 2003), we have identified a third unambiguous glitch that occured near MJD 53551 (2005 June 30). Note that the exact glitch epoch is unknown due to our noncontinuous monitoring; we report an epoch for which the phase jump is zero. This is because a non-zero phase jump at the time of the glitch would suggest an unphysically large torque on the star. This third glitch had fractional frequency jump $\Delta \nu / \nu=2.7 \times 10^{-6}$, and no obvious recovery. This glitch amplitude is intermediate between those of the previous two observed glitches, and the lack of recovery is similar to what was seen in the first glitch, but in marked contrast with the second glitch, as is clear from Figure 4, A sudden change in post-glitch spin-down rate for the third glitch is difficult to constrain, because of a possible additional glitch that occured not long after, as we describe below. Indeed glitch-induced long-term changes in $\dot{\nu}$ aside from that following the first glitch, as described by Kaspi et al. (2000), are difficult to identify given the apparent timing noise processes. Table 4 summarizes the parameters of the three certain glitches of RXS J170849.0-400910, assuming a glitch model consisting of a permanent change in $\nu$ and $\dot{\nu}$ and a frequency change $\nu_{d}$ that decayed on a time scale of $\tau_{d}$, i.e.,

$$
\nu=\nu_{0}(t)+\Delta \nu+\Delta \nu_{d} e^{-\left(t-t_{g}\right) / \tau_{d}}+\Delta \dot{\nu}\left(t-t_{g}\right)
$$

where $\nu_{0}(t)$ is the frequency evolution pre-glitch, $\Delta \nu$ is a instantaneous frequency jump, $\Delta \nu_{d}$ is the post-glitch frequency increase that decays exponentially on a time scale $\tau_{d}$, $t_{g}$ is the glitch epoch, and $\Delta \dot{\nu}$ is the post-glitch change in the long-term frequency derivative.

For the second glitch, residuals after subtraction of a simple glitch with fractional exponential recovery have clear remaining trends, as is clear in Figures 3 (second panel) and 4 (bottom panel). Systematic trends after simple glitch model subtraction were also reported by Woods et al. (2004) for the 2002 glitch in $1 \mathrm{E} 2259+586$. We also find this in the largest glitch in 1E 1841-045 (see $\S$ 3.2). Woods et al. (2004) showed that for $1 \mathrm{E} 2259+586$, the 
glitch fit was significantly improved by adding an exponential growth term. We have tried fitting this model to the second glitch from RXS J170849.0-400910 but find no improvement, with the preferred growth term consistent with zero.

In addition to the new certain glitch we report above, we find strong evidence for an additional three glitches, each having fractional amplitude similar to the first certain glitch seen in this source. The properties of these candidate glitches are summarized in Table 5 , Timing residuals around the epochs of these glitches are shown in Figure 5 , in the top panel. Residuals following the subtraction of a glitch model are shown in the middle panel of that Figure. We refer to these as candidates only because a 4-th order polynomial fit to the same data results in similar residuals (bottom panel of Fig. 5. Table 6) without the need to invoke a sudden event. The distinction between true glitches and timing noise is often difficult to make for small-amplitude glitches, as discussed by Kaspi et al. (2000). One way to distinguish, at least statistically, is that apparent discontinuities attributable to timing noise should not have a preferential direction, i.e., apparent spin-down 'glitches' should be seen too. An examination of the frequency panel in Figure 3 reveals apparent frequency jumps at the candidate glitch levels in both directions, suggesting one or more of the candidates could indeed be timing noise. Continued monitoring to acquire a larger database of such apparent discontinuities will help clarify this issue.

Subsequent to our submission and posting of this paper, Israel et al. (2007b) posted the results of a similar analysis of a subset of these same data. Some of their results are consistent with ours however others differ. They reported two glitches, the first of which corresponds to our second candidate glitch (Table 5). For that glitch, the reported fit parameters are similar though not identical to ours. Their second glitch corresponds to our third glitch in Table 4. For that glitch, the reported frequency jump at the glitch epoch was similar to ours but the jump in frequency derivative was significantly different. We find that this difference is due to their inclusion of more post-glitch TOAs when fitting the glitch. We did not include these TOAs because of a candidate event that occurs shortly thereafter, but which Israel et al. did not report. In addition to this difference, the frequency value reported in their post-glitch ephemeris, $0.09088624(2) \mathrm{Hz}$, is $42 \sigma$ away from the value $0.090885327(8) \mathrm{Hz}$ that we measure at the same epoch using our post-glitch ephemeris. The numbers in parentheses are $1 \sigma$ uncertainties. We do not understand this difference.

\subsection{Timing Results for $1 \mathrm{E} 1841-045$}

Figure 6 and Table 7 summarize the long-term timing behavior of 1E 1841-045. As for RXS J170849.0-400910, the spin evolution of 1E 1841-045 is well characterized by regular 
spin-down punctuated by occasional sudden spin-up events, plus timing noise. Ephemerides in Table 7 are given for the glitch-free intervals indicated in the top panel of Figure 6. As for RXS J170849.0-400910, the long-term timing residuals show some unmodelled trends whose origin is unknown. We consider these trends timing noise, as did Gotthelf et al. (2002) in their analysis of $\sim 2$ yr of data from this object. Note that the ephemeris in Table 7 labeled $\mathrm{B} 2$ is the same as that labeled B except for the omission of data immediately post-glitch (see caption to Fig. 6).

The frequency panel in Figure 6 and first panel in Figure 7 make clear that 1E 1841-045 suffered a large glitch, with significant recovery, near MJD 52460 (2002 July 5). This epoch is estimated, as for all glitches reported in this paper, by taking the epoch at which the phase jump is zero. Note that for this glitch there were several such epochs and the one we are reporting gives the most conservative frequency jump assuming an exponential recovery. The least conservative possible frequency jump is $\sim 50 \%$ larger. The glitch fractional amplitude was $\Delta \nu / \nu=1.6 \times 10^{-5}$ (see Table 8 ), among the largest yet seen from any neutron star. A fraction $Q \equiv \Delta \nu_{d} /\left(\Delta \nu_{d}+\Delta \nu\right)=0.64$ of the glitch recovered on a time scale of 43 days. This glitch is thus similar to the second certain glitch seen in RXS J170849.0-400910, and to the 2002 glitch in 1E 2259+586, which also showed significant recoveries on time scales of weeks. Also, like the second glitch of RXS J170849.0-400910, this large glitch in 1E 1841-045 is not well modelled by Equation 2, as is clear in the residuals plot in Figure 7 , Accompanying this frequency glitch was a substantial long-term increase in the the magnitude of $\dot{\nu}$, with fractional increase $\Delta \dot{\nu} / \dot{\nu}=0.0959 \pm 0.0007$. This is discussed further in $\$ 6.1$.

Because of the sparsity of the data around the glitch epoch, we found an alternate ephemeris for the period of time covered by ephemeris B (Fig. 6, Table 7). The fit parameters are $\nu=0.0849041677(17) \mathrm{Hz}, \dot{\nu}=-2.852(3) \times 10^{-13} \mathrm{~Hz} \mathrm{~s}^{-1}, \ddot{\nu}=-2.47(8) \times 10^{-21} \mathrm{~Hz} \mathrm{~s}^{-2}$, and $d^{3} \nu / d t^{3}=8.8(7) \times 10^{-29} \mathrm{~Hz} \mathrm{~s}^{-3}$ at the reported glitch epoch MJD 52464.00448, with RMS phase residual of 0.019. This ephemeris disagrees with ephemeris B in the shape of the recovery (see dotted curve in Panel 3 of Fig. 6) but agrees with it after the end of the recovery. Using the parameters of this alternate ephemeris, the change in $\nu$ at the glitch epoch would be $2.20(3) \times 10^{-7} \mathrm{~Hz}$ much smaller than the one reported in Table 8 , However, we hesitate to interpret the glitch using this ephemeris because of the very unusual and unique shape of the recovery it predicts. Note that this alternate ephemeris also shows a long-term increase in the magnitude of $\dot{\nu}$ after the glitch.

We also report the detection of two additional, smaller glitches, as summarized in Table 8 and displayed in Figures 6 and 7 . Neither glitch displays significant recovery, and both are well modelled by a simple permanent frequency jump. 


\section{Pulse Profile Changes}

Another interesting AXP property we can study thanks to RXTE monitoring is the evolution of the pulse profile. We performed a pulse profile analysis on each AXP using FTOOLS version 5.3.12. We used the following steps: for each observation, we ran the FTOOL make_se to combine the GoodXenon files. We then used the FTOOL fasebin to make a phase-resolved spectrum of the entire observation with 64 phase bins across the profile. When we ran fasebin, we selected layer 1 of the detector, disregarded the propane photons, and included the photons from PCUS 1, 2, 3, and 4. We omitted PCU 0, for which an independent analysis of AXP 4U 0142+61 revealed spectral modeling irregularities (Dib et al. 2007a). fasebin also took care of barycentering the data. For each observation, we then used seextrct to make a phase-averaged spectrum for the same set of detector layers and PCUs. The phase-averaged spectrum was then used by the perl script pcarsp to make a response matrix.

We loaded the phase-resolved spectra and the response matrices into the X-ray Spectral Fitting Package (XSPEC3) and selected photons belonging to three energy bands: 2-10, 2-4, and 4-10 keV. Using XSPEC, we extracted a count-rate pulse profile for each of the energy bands. The profiles included XSPEC-obtained $1 \sigma$ error bars on each of the phase bins. To obtain a pulse profile in units of count rate per PCU, we divided the overall profile by a PCU coverage factor that took into account the amount of time each PCU was on.

We then aligned the 64-bin profiles with a high signal-to-noise template using a similar cross-correlation procedure to the one used in the timing analysis. Then, for each glitch-free interval, we summed the aligned profiles, subtracted the DC component, and scaled the resulting profile so that the value of the highest bin is unity and the lowest point is zero.

\subsection{Profile Analysis results of RXS J170849.0-400910}

Average profiles for RXS J170849.0-400910 in the three energy bands are presented in Figure 8. In a given band, the different profile qualities are due to different net exposure times. Energy dependence is clearly visible to the eye as well as small fluctuations. For example, in the 2-4 keV band, the small peak off the main pulse has clearly fluctuating intensity. This small peak gets larger at higher energy, as seen in the $2-10 \mathrm{keV}$ band. In the 4-10 keV band, the smaller peak seems to blend with the main low-energy peak to yield a

\footnotetext{
${ }^{2}$ http://heasarc.gsfc.nasa.gov/ftools

${ }^{3}$ http://xspec.gsfc.nasa.gov Version: 11.3.1
} 
broad single peak structure, although fluctuations in that structure are apparent.

To study these fluctuations quantitatively, we subjected each profile to a Fourier analysis. Figure 9 shows the evolution of the first three profile harmonics with time. Although there are hints of variation in all energy bands, only variations in the hard 4-10 keV band are statistically significant (as determined by the $\chi^{2}$ statistic from a fit to a constant value); the decline of the second and third harmonics in the hard band have probabilities of $0.0007 \%$ and $0.0012 \%$, respectively, of being due to chance. Thus in the hard band the profile is certainly becoming more sinusoidal, in agreement with what is inferred by eye.

The above analysis shows that the profile is changing, but not whether these changes are truly correlated with the glitch epochs, since changes could be occuring throughout. To search for pulse profile changes correlated with glitch epochs, as were claimed by Dall'Osso et al. (2003), we divided glitch-free intervals into several sub-intervals (typically of duration $\sim 30$ days) for which independent profiles were created. The number of sub-intervals was chosen by trading off signal-to-noise ratio for time resolution. These sub-interval profiles were then Fourier analysed. The evolution of the Fourier powers in the first three harmonics in the 2-10 keV profile are shown in the top panel of Figure 10 .

To determine whether the apparent fluctuations are statistically significant, we fit a constant value to each time series and from the $\chi^{2}$ of the best fit, found that the probabilities of the fluctuations being due to random noise are 68,96 and $69 \%$ for $n=1,2,3$, respectively. This analysis thus shows no evidence for profile changes associated with the glitch epochs, including the second glitch. However the reduced signal-to-noise ratios in the sub-interval average profiles, required for interesting time resolution, makes us insensitive to subtle profile changes. To search for glitch-correlated pulse profile changes in a different way, for each subinterval we calculated the reduced $\chi^{2}$ of the difference between that sub-interval's average profile and the previous one. The time series of these $\chi^{2}$ values is shown in the bottom panel of Figure 10. There is clearly no evidence for any profile change at the second glitch, or at the third certain glitch. There is some hint of profile changes at the first and second candidate glitches, however a K-S test shows that our $\chi^{2}$ values as a group have a probability of $39 \%$ of originating from $\chi^{2}$ distribution. Interestingly though, the probability of the single high $\chi^{2}$ value we measure at the second candidate glitch occuring randomly is only $1.0 \times 10^{-6}$; that at the first glitch is $1.7 \%$ and at the first glitch candidate is $0.4 \%$. Thus we do find possible evidence in this analysis for glitch-correlated pulse profile changes, though the best evidence for significant changes occurs only at two candidate glitches, i.e., the lowest amplitude events. 


\subsection{Profile Analysis results of $1 \mathrm{E} 1841-045$}

Summed profiles for 1E 1841-045 in three energy bands for the five glitch-free intervals defined in the top panel of Figure 6 are shown in Figure 11. As for RXS J170849.0-400910, some low-level profile fluctuations are suggested, particularly in the relative amplitude of the leading and trailing sides of the large single peak in the 2-10 keV band (though clearly this peak could also be considered the blend of two or more adjacent peaks).

As for RXS J170849.0-400910, we quantify the profile fluctuations of 1E 1841-045 via Fourier analysis. Figure 12 shows the evolution of the first three profile harmonics with time in each energy band. Interestingly, in contrast to RXS J170849.0-400910, here the profile changes are most prominent in the soft $2-4 \mathrm{keV}$ band, in which the fraction of power in the fundamental of the profile in interval A2 (see Fig. 6) decreased, then slowly relaxed back to the previous range. However, a $\chi^{2}$ test shows the probability of this behavior being due to random noise is $18 \%$, too large to exclude.

To look for pulse profile changes correlated with glitches, again, sub-intervals within glitch-free intervals were chosen and summed profiles computed and Fourier analysed. The evolution of the first three harmonics is shown in the top panel of Figure 13. Fluctuations are apparent although none is particularly remarkable at any of the glitch epochs, including the first and largest, and the time series for $n=1,2,3$ are all consistent with a constant value. This argues again against correlated profile and timing anomalies in this source thus far. As a confirmation, as for RXS J170849.0-400910, a difference profile was calculated for each sub-interval by subtracting that interval's profile from the preceding one. The $\chi^{2}$ values of these difference profiles are shown in the bottom of Figure 13, no significant features are present.

\section{Pulsed Flux Time Series}

RXTE monitoring also allows the study of the evolution of the pulsed flux of these sources. To obtain a pulsed flux time series for RXS J170849.0-400910 and 1E 1841-045, we did the following. First, for each observation, we used a procedure similar to that described in $\$ 4$ to make a count rate per PCU pulse profile (with 64 phase bins across the profile and excluding PCU 0) in the energy range $2-10 \mathrm{keV}$. The profiles included XSPEC-determined $1 \sigma$ error bars on the flux value in each of the phase bins. 
The pulsed flux for each of the profiles was calculated using the following RMS formula:

$$
F=\sqrt{2 \sum_{k=1}^{n}\left(\left(a_{k}{ }^{2}+b_{k}{ }^{2}\right)-\left({\sigma_{a_{k}}}^{2}+{\sigma_{b_{k}}}^{2}\right)\right)},
$$

where $a_{k}$ is the $k^{\text {th }}$ even Fourier component defined as $a_{k}=\frac{1}{N} \sum_{i=1}^{N} p_{i} \cos (2 \pi k i / N), \sigma_{a_{k}}{ }^{2}$ is the uncertainty of $a_{k}, b_{k}$ is the odd $k^{\text {th }}$ Fourier component defined as $b_{k}=\frac{1}{N} \sum_{i=1}^{N} p_{i} \sin (2 \pi k i / N)$, $\sigma_{b_{k}}{ }^{2}$ is the uncertainty of $b_{k}, i$ refers to the phase bin, $N$ is the total number of phase bins, $p_{i}$ is the count rate in the $i^{\text {th }}$ phase bin of the pulse profile, and $n$ is the maximum number of Fourier harmonics to be taken into account. We used $n=6$ for both RXS J170849.0-400910 and 1E 1841-045.

Our method for estimating the pulsed flux $F$ is equivalent to the simple RMS formula $F=\frac{1}{\sqrt{N}} \sqrt{\sum_{i=1}^{N}\left(p_{i}-\bar{p}\right)^{2}}$ (where $p_{i}$ is the count rate in the $i^{\text {th }}$ phase bin of the pulse profile and $\bar{p}$ is the average count rate), except that we have subtracted the variances (to eliminate the upward statistical bias) and only included the statistically significant Fourier components. For a detailed discussion on pulsed flux estimates, see Archibald, Dib \& Kaspi (in prep.).

\subsection{Pulsed Flux Time Series for RXS J170849.0-400910}

Our pulsed flux time series for RXS J170849.0-400910 is shown in Figure 3 (bottom panel) and again in Figure 14. Each data point represents the average of pulsed fluxes measured over $\sim 1$ month. There appear to be frequent low-level pulse flux variations in this source. Although our error estimates on the pulsed fluxes include only statistical uncertainty (i.e., we have made no effort to estimate systematic uncertainties), we are given confidence that the fluctuations seen e.g., near MJD 52000 are real, given how stable the pulsed flux of $1 \mathrm{E} 1841-045$ is in the same time interval (see $\$ 5.2$ ).

There are no large increases in pulsed flux following any of the glitches, unlike what was seen following the 2002 glitch of AXP 1E 2259+586 (Kaspi et al. 2003; Woods et al. 2004). However, there is a possible pulsed flux enhancement prior to the second glitch, and a dip following it. Given this and the lack of clearly associated pulse profile changes coincident with glitch epochs (see 84.1 ), the glitches of RXS J170849.0-400910 appear to be "quiet," in the sense that they seem unaccompanied by significant pulsed radiative change. This is discussed further in $\$ 6$.

Figure 14 shows phase-averaged fluxes in the $0.5-10 \mathrm{keV}$ band as measured using a variety of focussing X-ray telescopes (Rea et al. 2005; Campana et al. 2007). Interestingly, while the reported phase-averaged flux varies considerably (by a factor of $\sim 1.6$ ), and in 
concert with the photon index as measured in the conventionally used blackbody/power-law spectral model, the $2-10 \mathrm{keV}$ pulsed flux remains relatively constant. This suggests that the pulsed fraction of RXS J170849.0-400910 is precisely anti-correlated with total flux, in such a way as to keep the pulsed flux near constant. This is discussed further in $₫ 6$.

The origin of the apparent low-level pulsed flux variations is not clear, given the apparent lack of correlation with the phase-averaged flux. As shown by Archibald et al. (in prep.), a changing pulse profile can affect an RMS-based pulsed flux estimator such as that in Equation 3. To verify that our measured pulsed fluxes were not influenced by the changing pulse profile of RXS J170849.0-400910 (see \$4), we also found the pulsed flux using an estimator based on the area under the profile (after baseline subtraction), which is, by definition, insensitive to pulse profile changes. With this method, we obtained qualitatively similar results for the pulsed fluxes.

\subsection{Pulsed Flux Time Series for 1E 1841-045}

Our pulsed flux time series for 1E 1841-045 is shown in the bottom panel of Figure 6. Each data point represents the average of pulsed fluxes measured over $\sim 1$ month. Note the increased scatter after MJD 52700 is due to decreased effective integration time, a result of the reduction in the average number of operational PCUs. The measured pulsed fluxes are consistent with being constant, with their probability of being due to random fluctuations $52 \%$. There is no evidence for any pulsed flux change at the glitch epochs. Thus the glitches of 1E 1841-045 appear to be "quiet," at least in pulsed flux, on time scales comparable to or longer than our sampling time.

\section{Discussion}

\subsection{AXP Glitches}

We have now observed a sufficiently large sample of AXP glitches that we can make meaningful phenomenological comparisons with glitches in radio pulsars, a much better studied phenomenon. Detection of systematic differences in AXP and radio pulsar glitch properties would be interesting as it could signal structural differences between magnetars and conventional radio pulsars.

Figure 15 shows the fractional and non-fractional amplitude distributions of radio pulsar and AXP glitches. As is clear from the figure, although the fractional glitch amplitudes of 
AXPs are generally large by radio pulsar standards, the AXP absolute glitch amplitudes, more directly related to the angular momentum transfer during the glitch, are neither especially large nor especially small. Thus, glitching in neutron stars is clearly not correlated with frequency as some studies of radio pulsars have suggested (Lyne et al. 2000).

Given the spectacular radiative outburst contemporaneous with the large 2002 1E 2259+586 glitch, we can speculate that larger angular momentum transfers that occur in radio pulsars could result in even more dramatic outbursts in affected AXPs, possibly like those seen in XTE 1810-197 (Ibrahim et al. 2004) and in the AXP candidate AX J1845-0258 (Tam et al. 2006). Indeed a recent X-ray burst observed from CXOU J164710.2-455216 (Muno et al. 2007) has been claimed to be accompanied by a very large $\left(\Delta \nu / \nu \simeq 6 \times 10^{-5}, \Delta \nu \simeq 6 \times 10^{-6}\right)$ glitch (Israel et al. 2007a), and AXP 1E 1048.1-5937 recently exhibited a large glitch and flux increase (Dib et al. 2007b, Dib et al., in prep.). However the lack of any observed radiative change in 1E 1841-045 around the time of its first observed glitch, which was over a factor of two larger than that in 1E $2259+586$ in terms of absolute frequency jump, argues against this idea. Clearly, the data are indicating that AXP glitches, even large ones, can be either radiatively loud or quiet.

Glitch activity has been defined as

$$
a_{g}=\frac{1}{\Delta t} \sum \frac{\Delta \nu}{\nu}
$$

where $\Delta t$ is the total observing span and the sum is over all glitches, and includes decaying components (McKenna \& Lyne 1990). We refer to $a_{g}$ as fractional activity, since it involves the sum of fractional frequency changes. One can also define an absolute glitch activity,

$$
A_{g}=\frac{1}{\Delta t} \sum \Delta \nu
$$

where the sum is over the absolute frequency changes (e.g., Wang et al. 2000). The quantities $a_{g}$ and $A_{g}$, introduced for the study of radio pulsars, are approximately interchangeable for those objects, given that the range of frequencies encompassed by glitching radio pulsars is relatively small. By contrast, when considering AXPs and their much smaller rotation frequencies, a comparison with radio pulsars for $a_{g}$ and $A_{g}$ are very different (see, e.g., Heyl \& Hernquist 1999). Also, for establishing the average amount of spin-up imparted to the crust over time, the total frequency increase at each epoch is relevant. However, in some instances, the quantity of interest is the unrelaxed portion of the glitch, i.e., the permanent frequency jump only. In general, for radio pulsars, $Q$ is small so this distinction is not important. However for AXPs, given the paucity of glitches we have observed thus far as well as the fact that several, particularly the largest, of these have had large values of $Q$ (e.g., $Q \simeq 1$ for the second glitch seen in RXS J170849.0-400910), the distinction between 
including the total frequency jump and only the unrelaxed frequency jump is important. We choose here to remain with convention and include the total frequency jump at each epoch when calculating $a_{g}$ and $A_{g}$, although this choice should be kept in mind.

With 8.7 yr of monitoring of RXS J170849.0-400910, we can now reasonably calculate glitch activity parameters for this source using Equations 4 and 5. If only counting the unambiguous glitches, $a_{g}=2.9 \times 10^{-14} \mathrm{~s}^{-1}$ and $A_{g}=2.5 \times 10^{-15} \mathrm{~s}^{-2}$. Including candidate glitches only increases these numbers by $\sim 20 \%$. With three glitches in $7.6 \mathrm{yr}, 1 \mathrm{E} 1841-045$ is evidently a very active glitcher as well. Its glitch activity parameters are $a_{g}=7.9 \times 10^{-14} \mathrm{~s}^{-1}$ and $A_{g}=6.7 \times 10^{-15} \mathrm{~s}^{-2}$. Indeed $A_{g}$ for $1 \mathrm{E} 1841-045$ is the highest glitch activity seen thus far in any neutron star, radio pulsar or AXP, to our knowledge. We also calculated a tentative glitch activity for AXP 1E $2259+586$, for which we have observed two glitches, the well documented one in 2002 (Kaspi et al. 2003; Woods et al. 2004) and a second, smaller glitch that occured very recently and had fractional amplitude $8.5 \times 10^{-7}$ and no recovery (Dib et al., in prep.). Using these events and given that we have observed this source with $R X T E$ for $9.4 \mathrm{yr}$, we find $a_{g}=1.7 \times 10^{-14} \mathrm{~s}^{-1}$ and $A_{g}=2.4 \times 10^{-15} \mathrm{~s}^{-1}$.

We can plot these activities as a function of pulsar age (as estimated via spin-down age $\nu / 2 \dot{\nu})$ and $\dot{\nu}$; see Figure 16. Previous authors have noted interesting correlations on these plots for radio pulsars (e.g., Lyne et al. 2000; Wang et al. 2000); these are seen in our plots as well. Note that upper limits for some radio pulsars of relevant ages fall well below the apparent correlations (e.g., Wang et al. 2000); we choose not to plot those because, as discussed in that reference, a single glitch of average size would bring them roughly in line with the correlation. Note that the radio pulsar outlier at small age and high $\dot{\nu}$ in all plots is the Crab pulsar, long-known to exhibit few and small glitches. We also looked for a trend in a plot of $a_{g}$ or $A_{g}$ versus surface dipolar field (as estimated via $3.2 \times 10^{19} \sqrt{P \dot{P}} \mathrm{G}$ ) but found none.

As a group, the AXPs do not especially distinguish themselves when either activity, $a_{g}$ or $A_{g}$ is plotted versus spin-down age, though they do increase the scatter. This suggests a universal correlation with spin-down age. The same is true of $A_{g}$ plotted versus $\dot{\nu}$. However, interestingly, the AXPs as a group all stand out on the diagram of $a_{g}$ versus $\dot{\nu}$ (Fig. 16), such that for similar spin-down rates, their fractional glitch activities are much larger than in radio pulsars.

Link et al. (1999) argued that $a_{g}$ provides a strict lower limit on the fraction of the moment of inertia of the neutron star that resides in the angular momentum reservoir (generally assumed to be the crustal superfluid) tapped during spin-up glitches, $I_{\text {res }}$. They showed that $I_{\text {res }} / I_{c} \geq \nu a_{g} /|\dot{\nu}| \equiv G$, where $I_{c}$ is the moment of inertia of the crust and all components strongly coupled to it, and $G$ is a "coupling parameter." For radio pulsars, they argued 
for a universal $G$ that implies $I_{\text {res }} / I_{c} \geq 0.014$. It is interesting to ask whether this same apparently universal relationship holds for AXPs. Figure [17 shows $G$ plotted versus age for radio pulsars and AXPs. As is clear, the Link et al. (1999) relation seems to hold for the radio pulsars, even with increased glitch statistics. Also, AXPs RXS J170849.0-400910 and 1E 1841-045 lie among the radio pulsars, suggesting similar reservoir fractions. However the outlier point, $1 \mathrm{E} 2259+586$, has $G=0.25$, much larger than the others. Admittedly, for this AXP, $a_{g}$ is estimated from two glitches only, with the 2002 event greatly dominating, so the values are tentative. Still, the large $G$, if real, suggests that at least $\sim 25 \%$ of the stellar moment of inertia is in the angular momentum reservoir (see also Woods et al. 2004). We note that the analysis of Link et al. (1999) ignores recovery, important for the $20021 \mathrm{E} 2259+586$ glitch, which dominates its $a_{g}$. However in the 2002 glitch, the recovery fraction was only $\sim 19 \%$, so even accounting for recovery, $G$ for $1 \mathrm{E} 2259+586$ is surprisingly high.

As described by Kaspi et al. (2003) and Woods et al. (2004), the $20021 \mathrm{E} \mathrm{2259+586}$ glitch was unusual when compared with those of radio pulsars. Specifically the combination of the recovery time scale and the large recovery fraction $Q$ conspired to make the pulsar spin down, for over two weeks post-glitch, at over twice its long-term average spin-down rate. Although spin-down rate enhancements post-glitch are often seen in radio pulsars (e.g., Flanagan 1990), they usually amount to only a few percent. A remarkably large post-glitch spin-down rate enhancement was seen also in the second glitch of RXS J170849.0-400910 and the first observed glitch of $1 \mathrm{E}$ 1841-045, though to a lesser degree than in 1E 2259+586. Of course a much larger increase in spin-down rate post-glitch in RXS J170849.0-400910 or 1E 1841-045 could have been missed due to our sparse sampling.

One way to quantify the enhanced spin-down more precisely is using Equation 2 at $t=0$, and noticing that the instantaneous spin-down rate at the glitch epoch due to the exponential recovery is given by $\Delta \nu_{d} / \tau$. Comparing this quantity for the AXP glitches that show recovery with the pre-glitch time-averaged spin-down rate $\dot{\nu}$, we find that for $1 \mathrm{E} 2259+586 \Delta \nu_{d} / \tau=(8.2 \pm 0.6) \dot{\nu}, \Delta \nu_{d} / \tau=(0.64 \pm 0.6) \dot{\nu}$ for RXS J170849.0-400910, and $\Delta \nu_{d} / \tau=(0.75 \pm 0.08) \dot{\nu}$ for $1 \mathrm{E} 1841-045$, all very large by radio pulsar standards.

The increase in spin-down rate post-glitch, at least for radio pulsars, is generally attributed to a decoupling of a small percentage of the moment of inertia of the star, usually presumed to be part of the crustal superfluid (e.g., Pines \& Alpar 1985), with constant external torque. If the observed AXP recoveries and temporarily enhanced spin-down rates were interpreted in the same way, it would imply that very large fractions (ranging from 0.4 to 0.9) of the moment of inertia of the star decoupled at the glitch, much larger than the crustal superfluid is reasonably expected to comprise, for any interior equation of state. To avoid this 
problem, Woods et al. (2004) suggested that a pre-glitch rotational lag between the crust and superfluid might have temporarily reversed at the glitch (see also Alpar et al. 2000). Then the observed larger spin-down rate post-glitch would be due to the crust transferring angular momentum back to the superfluid in order to reestablish equilibrium.

In glitches, the equilibrium angular velocity lag between the crust and more rapidly rotating crustal superfluid is thought to be the origin of glitches. This lag is proposed to develop because the crustal superfluid's angular momentum vortices, in many models (e.g., Alpar et al. 1984a), become pinned to crustal nuclei and hence are hindered from moving outward as the star's crust and associated components are slowed by the external torque. How this lag could reverse is puzzling. Woods et al. (2004) suggested that a twist of magnitude $10^{-2}$ rad of a circular patch of crust offset in azimuth from the rotation axis could result in sufficient spin-down of the crustal superfluid to account for the properties of the 2002 glitch in $1 \mathrm{E} 2259+586$. They noted further that such a twist also produces X-rays of the luminosity observed in that outburst (Thompson et al. 2002). The absence of any significant radiative changes at the time of largest glitches in RXS J170849.0-400910 and 1E 1841-045 is thus problematic for the crustal twist, and hence lag reversal, model. We note that it has been argued independently that a similar suggested lag reversal between crust and crustal superfluid in the Vela pulsar is unphysical (Jahan-Miri 2005).

We also note that the large and long-term increase in the magnitude of $\dot{\nu}$ following the large glitch in 1E 1841-045 (see §3.2) is also interesting. Alpar et al. (1993) showed that, ignoring transient terms, $I_{r e s} / I_{c} \geq \Delta \dot{\nu} / \dot{\nu}$. For the large 1E 1841-045 glitch, this implies $I_{\text {res }} / I_{c} \geq 0.1$, much larger than has been seen in any radio pulsar.

One possibility that can explain the large $G$ for $1 \mathrm{E} 2259+586$, the large transient increases in the magnitude of $\dot{\nu}$ in all three large glitches, as well as the large extended $\dot{\nu}$ change in the first 1E 1841-045 glitch, is that core superfluid is somehow involved, as it is expected to carry the bulk of the moment of inertia. We note that core glitches have been discussed in the radio pulsar context for some time, albeit for very different reasons. Although Alpar et al. (1984b) argued that the crust and core should be strongly coupled on very short time scales, Jones (1998) found that crustal pinning of superfluid vortices cannot be occuring in neutron stars, because the maximum pinning force is orders of magnitude smaller than the estimated vortex Magnus force. Donati \& Pizzochero (2003) argue that crustal vortex pinning cannot occur for independent reasons. If these authors are correct, pulsar glitches would generally not originate in the crust.

Why would the clearest evidence for core glitches come from AXPs? The interaction between vortices and quantized magnetic flux tubes in a core superfluid could provide resistance to outward motion of vortices (Jones 1998; Ruderman et al. 1998; Jones 2002). The 
interior magnetic field playing a role in vortex pinning as studied by Ruderman et al. (1998) could help explain why such unusual glitch recoveries are seen preferentially in AXPs, which appear to have much larger magnetic fields than conventional radio pulsars. Perhaps the larger field, which implies a higher density of flux tubes, can effectively pin more superfluid vortex lines in a magnetar core; with greater magnetic activity, sudden magnetic reconfigurations would result in large core vortex reconfigurations. We note that Kaspi et al. (2000) and Dall'Osso et al. (2003) argued that the Ruderman et al. (1998) model must be inapplicable to AXPs as that model predicts no glitches for periods greater than $\sim 0.7 \mathrm{~s}$. However a more careful reading of Ruderman et al. (1998) reveals that this prediction does not apply for magnetar-strength magnetic fields.

As pointed out by Kaspi et al. (2000), the high temperatures of AXPs, as measured from their X-ray spectra, are at odds with the glitch observations. This is because in the crustal pinning models, the pinning force is highly temperature dependent, such that vortex lines can creep outward much more easily when the temperature is high (e.g., Alpar et al. 1989). This has long been the explanation (e.g., Anderson \& Itoh 1975) for the difference between the Crab and Vela pulsar glitch behaviors: the hotter Crab pulsar glitches less frequently and with smaller frequency jumps because its vortex array can move outward more smoothly. If this were true, AXPs, having measured effective temperatures much higher than the Crab pulsar, should glitch less frequently and with smaller glitch amplitudes than the Crab pulsar, clearly not what is observed. If we abandon the crustal glitch model (at least in AXP glitches) the absence of the expected temperature dependence and the observed universal age correlation could be explained. For example, as discussed by Jones (1998), perhaps relatively smooth outward motion of core vortices could take place before the magnetic flux distribution necessary for impeding them has developed. In this picture, magnetic field distribution, not temperature, is the age-associated property that is a primary factor determining glitch behavior.

Finally, Campana et al. (2007) argued, on the basis of seven observations of RXS J170849.0-400910 obtained over $\sim 10 \mathrm{yr}$, that observed spectral and flux variations were correlated with glitch epoch. They predict, given apparent flux increases seen in mid-2004 and mid-2005, that a glitch should occur soon thereafter (see Fig. 14). Indeed as we have shown (see Table 4), an unambiguous glitch occured just following their mid-2005 observation. On the other hand, the first candidate glitch (Table 5) occured when the total flux was very low and apparently declining. Thus, if there is a causal connection between long-term flux variations in RXS J170849.0-400910 and glitches, either this candidate glitch is not a true glitch, or accompanying radiative changes are only relevant to large glitches. The sparsity of the total flux measurements, along with the relatively short time scale of the pulsed flux variations we report in RXS J170849.0-400910, suggest that more dense total flux monitoring 
could reveal yet unseen fluctuations that are not glitch-associated. The flux variability of RXS J170849.0-400910 is discussed further below.

\subsection{Radiative Changes}

The approximate stabilities of the pulsed fluxes of RXS J170849.0-400910 and 1E 1841-045 (see 95) are in contrast to those seen for AXPs 1E 2259+586 and 1E 1048.1-5937, both of which have shown large pulsed flux variations (Kaspi et al. 2003; Woods et al. 2004; Gavriil \& Kaspi 2004), and even 4U 0142+61 which has shown a slow pulsed flux increase with time (Dib et al. 2007a). It is interesting that the phase-averaged flux of RXS J170849.0-400910 has been reported to be highly variable (Rea et al. 2005; Campana et al. 2007 and Fig. 14), with changes as large as $\sim 60 \%$ in 2004-2005, while the pulsed flux is not, with maximum contemporaneous change of $<15 \%$. Note that this conclusion holds even when the phaseaveraged fluxes, reported in the $0.5-10 \mathrm{keV}$ band, are converted to $2-10 \mathrm{keV}$, that used for our RXTE observations. This suggests an anti-correlation between pulsed fraction and total flux that acts to ensure that the pulsed flux is roughly constant. If so, pulsed flux is not a good indicator of total energy output for RXS J170849.0-400910 $\dot{A}$ similar anticorrelation between total flux and pulsed fraction has been reported for AXP 1E 1048-5937 (Tiengo et al. 2005; Gavriil et al. 2006; Gavriil et al. 2007), although in that case the pulsed flux does not remain constant (Gavriil \& Kaspi 2004), but follows the phase-averaged flux, just with lower dynamic range (Tam et al., submitted). For RXS J170849.0-400910, the exactness of the anti-correlation is perhaps surprising. It could be that all the phase-averaged flux variations are in the $0.5-2 \mathrm{keV}$ band, invisible to $R X T E$. However this would not jibe

with the reported correlation of the phase-averaged fluxes with power-law index (Rea et al. 2005; Campana et al. 2007). We also note that in the phase-averaged flux analysis, the equivalent hydrogen column $N_{H}$ was allowed to vary from observation to observation, rather than being held fixed at a constant. This inconsistency could bias the comparison, though likely not by a large amount. It is tempting to question the relative calibrations of the different instruments used to measure the phase-averaged flux of RXS J170849.0-400910, as the greatest dynamic range is implied by lone XMM-Newton and Swift observations, and even the two Chandra X-ray Observatory observations were obtained with different instruments. Still, admittedly, relative systematic calibration uncertainties are not expected to yield a $>50 \%$ dynamic range, as is reported. Regular monitoring with a single imaging instrument could settle this issue.

Changes in pulse profile seem to be generic in AXPs and, as discussed above, are not always correlated with glitches, although in some cases, e.g., 1E 2259+586, 4U 0142+61, 
and possibly RXS J170849.0-400910, they are. Spectrally no clear pattern has emerged. In $1 \mathrm{E} 2259+586$, the pulse profile changes following its 2002 event were broadband (Woods et al. 2004), while in 4U 0142+61 (Dib et al. 2007a) and 1E 1841-045, they appear more prominent at soft energies. In RXS J170849.0-400910, the changes are more apparent in the hard band. In the context of the magnetar model, this hints at crustal motions and surface activity, possibly coupled with magnetospheric activity, with the exact observational manifestation dependent on a variety of factors ranging from viewing geometry to magnetospheric scattering optical depth. Whether ultimately this specific phenomenon will provide insights into the physics of magnetars remains to be seen.

\section{Summary}

We have reported on long-term RXTE monitoring of AXPs RXS J170849.0-400910 and 1E 1841-045, which has allowed us to study these sources' timing, pulsed flux, and pulse profile evolutions.

We have discovered four new AXP spin-up glitches, one in RXS J170849.0-400910 and three in 1E 1841-045, plus three new glitch candidates in RXS J170849.0-400910. Nearly all of the "classical" AXPs have now been seen to glitch, clearly demonstrating that this behavior is generic to the class. Moreover, in terms of fractional frequency increases, AXPs are among the most actively glitching neutron stars known. Further, unlike radio pulsar glitches, AXP glitches appear to come in two varieties: those that, like radio pulsars, are radiatively quiet in pulsed flux, and those that are, unlike radio pulsars, radiatively loud, including correlated sudden flux increases and pulse profile changes. Thus far there is no clear correlation between AXP glitch size and whether or not it will be radiatively loud or quiet two of the largest AXP glitches thus far were quiet. We have found a substantial long-term increase in the magnitude of the spin-down rate in the largest glitch from 1E 1841-045, and have also shown that large AXP glitches often have recoveries that are unusual compared with those seen in radio pulsars. Specifically, their spin-down rates in the days and sometimes weeks after a glitch are significantly larger in absolute value than their long-term spin-down rate. This latter effect may indicate a temporary reversal in the crust/crustal superfluid lag at the time of the glitch, or possibly more plausibly, and certainly more intriguingly, glitches of the core, which could explain the transient and extended $\dot{\nu}$ increases, as well as the large $G$ value for $1 \mathrm{E} 2259+586$.

Radiatively, we have found that the pulsed fluxes of RXS J170849.0-400910 and 1E 1841-045 are both fairly steady with time. This is perhaps surprising in light of the large changes in phase-averaged flux that have been reported for RXS J170849.0-400910, and suggests, un- 
less the latter are affected by systematic calibration uncertainties, that pulsed flux for this source, as for AXP 1E 1048.1-5937 (Tiengo et al. 2005), is not a good indicator of AXP X-ray output. Also, the pulse profiles of RXS J170849.0-400910 and 1E 1841-045 both evolve; such evolution appears also to be a generic property of AXPs. However, no clear patterns in AXP pulse profile changes have yet emerged beyond occasional correlation with glitches. Hopefully further monitoring will shed physical light on this phenomenon.

We are grateful to Andrew Lyne for providing his unpublished glitch catalog. We thank Andrew Cumming, Chris Thompson and Pete Woods for useful comments. This work was supported by the Natural Sciences and Engineering Research Council (NSERC) PGSD scholarship to RD. FPG is supported by the NASA Postdoctoral Program administered by Oak Ridge Associated Universities at NASA Goddard Space Flight Center. Additional support was provided by NSERC Discovery Grant Rgpin 228738-03, NSERC Steacie Supplement Smfsu 268264-03, FQRNT, cifar, and CFI. VMK holds the Lorne Trottier in Astrophysics and Cosmology and a Canada Research Chair in Observational Astrophysics.

\section{REFERENCES}

Alpar, M. A., Anderson, P. W., Pines, D., \& Shaham, J. 1984a, ApJ, 278, 791

Alpar, M. A., Chau, H. F., Cheng, K. S., \& Pines, D. 1993, ApJ, 409, 345

Alpar, M. A., Cheng, K. S., \& Pines, D. 1989, ApJ, 346, 823

Alpar, M. A., Langer, S. A., \& Sauls, J. A. 1984b, ApJ, 282, 533

Alpar, M. A., Pines, D., \& Cheng, K. S. 2000, Nature, 348, 707

Anderson, P. W. \& Itoh, N. 1975, Nature, 256, 25

Arzoumanian, Z., Nice, D. J., Taylor, J. H., \& Thorsett, S. E. 1994, ApJ, 422, 671

Camilo, F., Ransom, S., Halpern, J., Reynolds, J., Helfand, D., Zimmerman, N., \& Sarkissian, J. 2006, Nature, 442, 892

Campana, S., Rea, N., Israel, G. L., Turolla, R., \& Zane, S. 2007, A\&A, 463, 1047

Dall'Osso, S., Israel, G. L., Stella, L., Possenti, A., \& Perozzi, E. 2003, ApJ, 599, 485

Dhillon, V. S., Marsh, T. R., Hulleman, F., van Kerkwijk, M. H., Shearer, A., Littlefair, S. P., Gavriil, F. P., \& Kaspi, V. M. 2005, MNRAS, 363, 609 
Dib, R., Kaspi, V. M., \& Gavriil, F. P. 2007a, ApJ, 666, 1152

Dib, R., Kaspi, V. M., Gavriil, F. P., \& Woods, P. M. 2007b, ATEL 1041

Donati, P. \& Pizzochero, P. M. 2003, Phys. Rev. Lett., 90, 211101+

Durant, M. \& van Kerkwijk, M. H. 2005, ApJ, 627, 376

Flanagan, C. S. 1990, Nature, 345, 416

Gavriil, F. P. \& Kaspi, V. M. 2004, ApJ, 609, L67

Gavriil, F. P., Kaspi, V. M., \& Woods, P. M. 2002, Nature, 419, 142

-. 2004, ApJ, 607, 959

Gavriil, F. P., Kaspi, V. M., \& Woods, P. M. 2006, ApJ, 641, 418

Gavriil, F. P., Kaspi, V. M., Woods, P. M., \& Dib, R. 2007, ATEL 1076

Gonzalez, M. E., Dib, R., Kaspi, V. M., Woods, P. M., Tam, C. R., \& Gavriil, F. P. 2007, submitted; (astro-ph/0708.2756)

Gotthelf, E. V., Gavriil, F. P., Kaspi, V. M., Vasisht, G., \& Chakrabarty, D. 2002, ApJ, 564, L31

Heyl, J. S. \& Hernquist, L. 1999, MNRAS, 304, L37

Hobbs, G., Lyne, A. G., Kramer, M., Martin, C. E., \& Jordan, C. 2004, MNRAS, 353, 1311

Hulleman, F., van Kerkwijk, M. H., \& Kulkarni, S. R. 2004, A\&A, 416, 1037

Ibrahim, A. I., Markwardt, C. B., Swank, J. H., Ransom, S., Roberts, M., Kaspi, V., Woods, P. M., Safi-Harb, S., Balman, S., Parke, W. C., Kouveliotou, C., Hurley, K., \& Cline, T. 2004, ApJ, 609, L21

Israel, G. L., Campana, S., Dall'Osso, S., Muno, M. P., Cummings, J., Perna, R., \& Stella, L. 2007a, ApJ, 664, 448

Israel, G. L., Gotz, D., Zane, S., Dall'Osso, S., Rea, N., \& Stella, L. 2007b, (astroph/0707.0485v2)

Israel, G. L., Covino, S., Stella, L., Campana, S., Marconi, G., Mereghetti, S., Mignani, R., Negueruela, I., Oosterbroek, T., Parmar, A. N., Burderi, L., \& Angelini, L. 2002, ApJ, 580, L143 
Jahan-Miri, M. 2005, New Astronomy, 11, 157

Jahoda, K., Swank, J. H., Giles, A. B., Stark, M. J., Strohmayer, T., Zhang, W., \& Morgan, E. H. 1996, Proc. SPIE, 2808, 59

Jones, P. B. 1998, MNRAS, 296, 217

—. 2002, MNRAS, 335, 733

Kaspi, V. M. 2007, Ap\&SS, in press (astro-ph/0610304)

Kaspi, V. M., Chakrabarty, D., \& Steinberger, J. 1999, ApJ, 525, L33

Kaspi, V. M. \& Gavriil, F. P. 2003, ApJ, 596, L71

Kaspi, V. M., Gavriil, F. P., Woods, P. M., Jensen, J. B., Roberts, M. S. E., \& Chakrabarty, D. 2003, ApJ, 588, L93

Kaspi, V. M., Lackey, J. R., \& Chakrabarty, D. 2000, ApJ, 537, L31

Kern, B. \& Martin, C. 2002, Nature, 415, 527

Kuiper, L., Hermsen, W., den Hartog, P., \& Collmar, W. 2006, ApJ, 645, 556

Link, B., Epstein, R. I., \& Lattimer, J. M. 1999, Phys. Rev. Lett., 83, 3362

Livingstone, M. A., Kaspi, V. M., Gavriil, F. P., \& Manchester, R. N. 2005, ApJ, 619, 1046

Lyne, A. G., Shemar, S. L., \& Graham-Smith, F. 2000, MNRAS, 315, 534

McKenna, J. \& Lyne, A. G. 1990, Nature, 343, 349

Mereghetti, S., Israel, G. L., \& Stella, L. 1998, MNRAS, 296, 689

Muno, M. P., Gaensler, B. M., Clark, J. S., de Grijs, R., Pooley, D., Stevens, I. R., \& Portegies Zwart, S. F. 2007, MNRAS, 378, L44

Özel, F. \& Guver, T. 2007, ApJ, 659, L141

Pines, D. \& Alpar, M. A. 1985, Nature, 316, 27

Rea, N., Israel, G. L., Testa, V., Stella, L., Mereghetti, S., Tiengo, A., Oosterbroek, T., Mangano, V., Campana, S., Covino, S., Curto, G. L., \& Perna, R. 2004, The Astronomer's Telegram, 284 
Rea, N., Oosterbroek, T., Zane, S., Turolla, R., Méndez, M., Israel, G. L., Stella, L., \& Haberl, F. 2005, MNRAS, 361, 710

Ruderman, M., Zhu, T., \& Chen, K. 1998, ApJ, 492, 267

Tam, C. R., Gavriil, F. P., Dib, R., Kaspi, V. M., Woods, P. M., \& Bassa, C., submitted; (astro-ph/0707.2093)

Tam, C. R., Kaspi, V. M., Gaensler, B. M., \& Gotthelf, E. V. 2006, ApJ, 652, 548

Tam, C. R., Kaspi, V. M., van Kerkwijk, M. H., \& Durant, M. 2004, ApJ, 617, L53

Thompson, C. \& Duncan, R. C. 1996, ApJ, 473, 322

Thompson, C., Lyutikov, M., \& Kulkarni, S. R. 2002, ApJ, 574, 332

Tiengo, A., Mereghetti, S., Turolla, R., Zane, S., Rea, N., Stella, L., \& Israel, G. L. 2005, A\&A, 437, 997

Wang, N., Manchester, R. N., Pace, R., Bailes, M., Kaspi, V. M., Stappers, B. W., \& Lyne, A. G. 2000, MNRAS, 317, 843

Wang, Z. \& Chakrabarty, D. 2002, ApJ, 579, L33

Wang, Z., Chakrabarty, D., \& Kaplan, D. L. 2006, Nature, 440, 772

Wilson, C. A., Dieters, S., Finger, M. H., Scott, D. M., \& van Paradijs, J. 1999, ApJ, 513, 464

Woods, P. M., Kaspi, V. M., Thompson, C., Gavriil, F. P., Marshall, H. L., Chakrabarty, D., Flanagan, K., Heyl, J., \& Hernquist, L. 2004, ApJ, 605, 378

Woods, P. M., Kouveliotou, C., Gavriil, F. P., Kaspi, M., V., Roberts, M. S. E., Ibrahim, A., Markwardt, C. B., Swank, J. H., \& Finger, M. H. 2005, ApJ, 629, 985

Woods, P. M. \& Thompson, C. 2006, in Compact Stellar X-ray Sources, ed. W. H. G. Lewin \& M. van der Klis (UK: Cambridge University Press) 


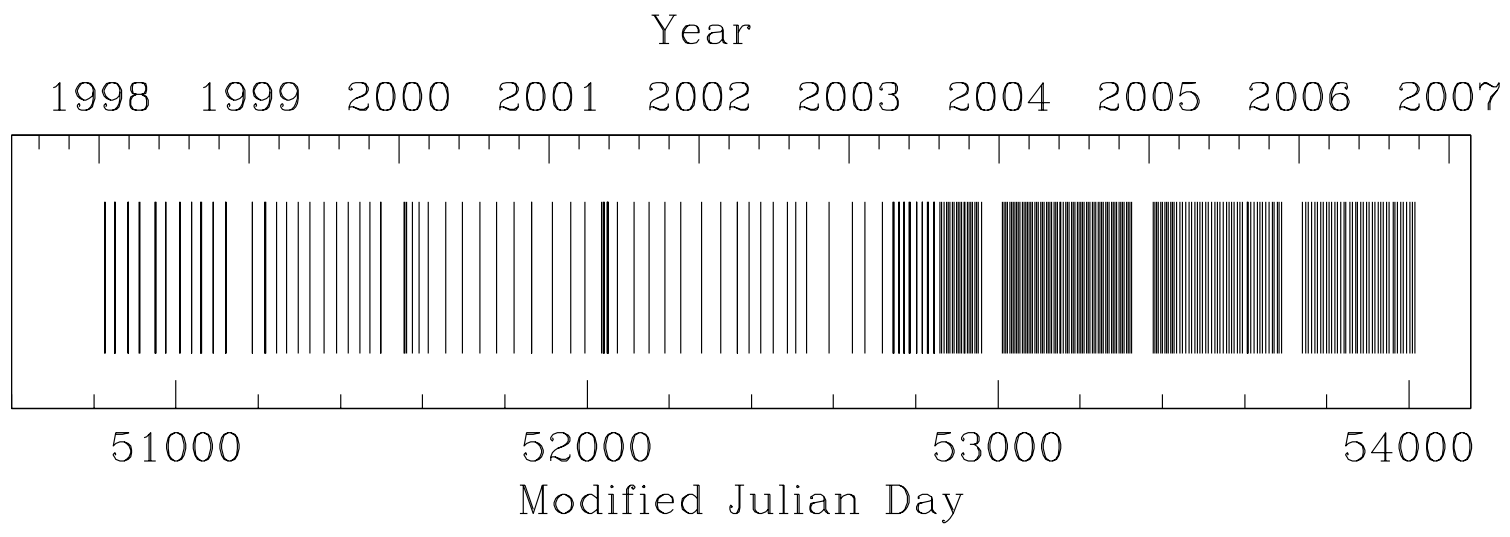

Fig. 1.- Epochs of observations of RXS J170849.0-400910 with RXTE. Gaps near the end/start of each year are due to Sun avoidance. See Table 1 for details. 


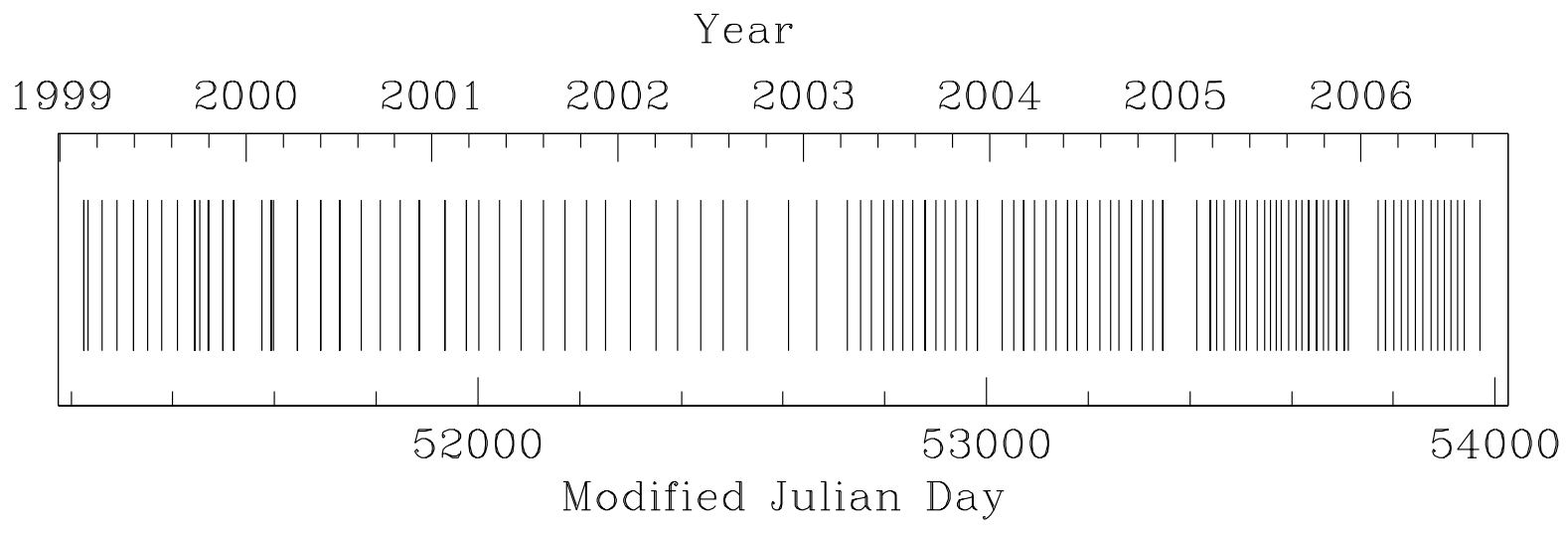

Fig. 2.- Epochs of observations of 1E 1841-045 with RXTE. Gaps near the end/start of each year are due to Sun avoidance. See Table 2 for details. 


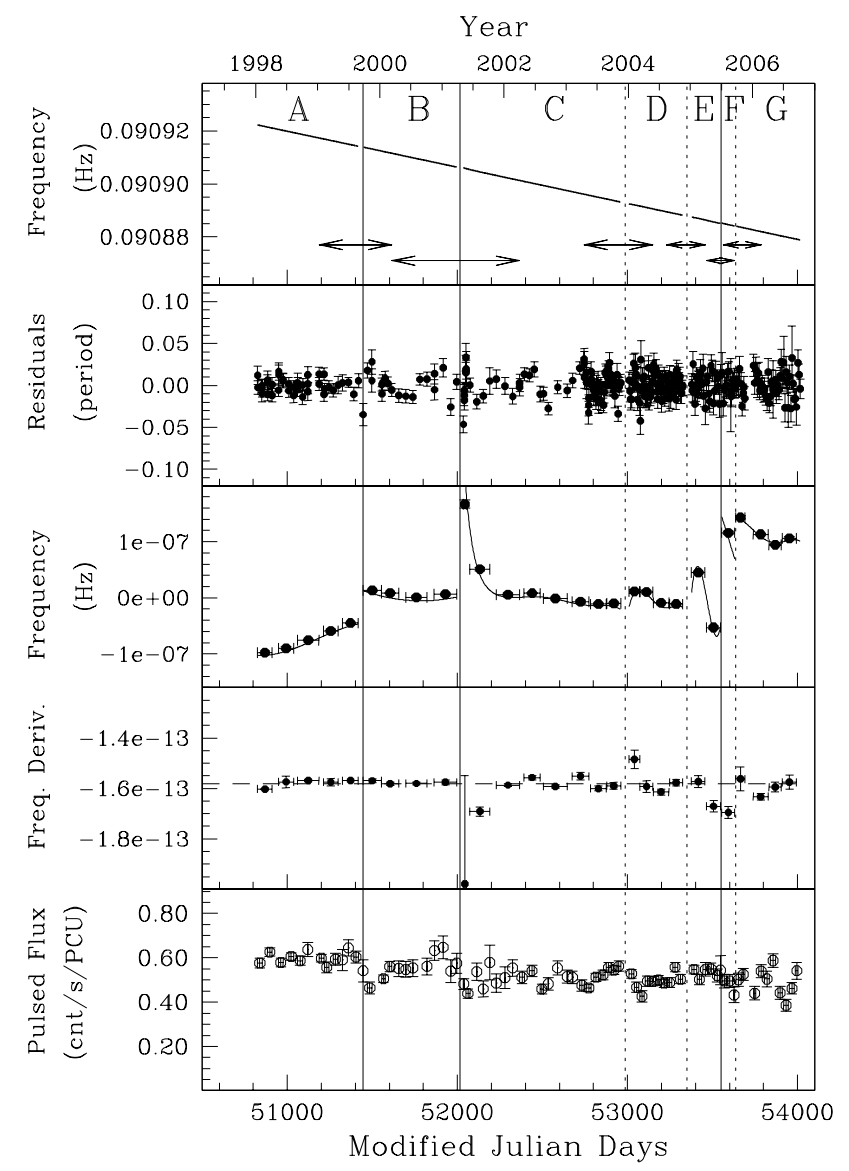

Fig. 3.- Spin and pulsed flux evolution in RXS J170849.0-400910. Panels are described from top to bottom. Top: Frequency evolution, with inter-glitch intervals indicated for correspondence with ephemerides given in Table 3. Arrows indicate intervals for which glitch ephemerides were obtained (see Table 4). Next: residuals, after subtraction of the best-fit models given in Table 3 (with arbitrary inter-interval phase offsets subtracted). The increased scatter after MJD 52600 is due to a decrease in typical integration time and an increase in monitoring frequency. Next: Solid curve: frequency evolution of the models shown in Table 3 after removal of the linear trend defined by the frequency and frequency derivative from interval $\mathrm{C}$ as measured by fitting only those parameters. Data points: measured frequencies in independent sub-intervals after subtraction of the extrapolation of the same linear trend. Next: Evolution of the frequency derivative in sub-intervals, when fitting locally for only $\nu$ and $\dot{\nu}$. Bottom: Pulsed flux in the $2-10 \mathrm{keV}$ range. All panels: Unambiguous glitch epochs are indicated with solid vertical lines. Candidate glitch epochs are indicated with dashed vertical lines. 


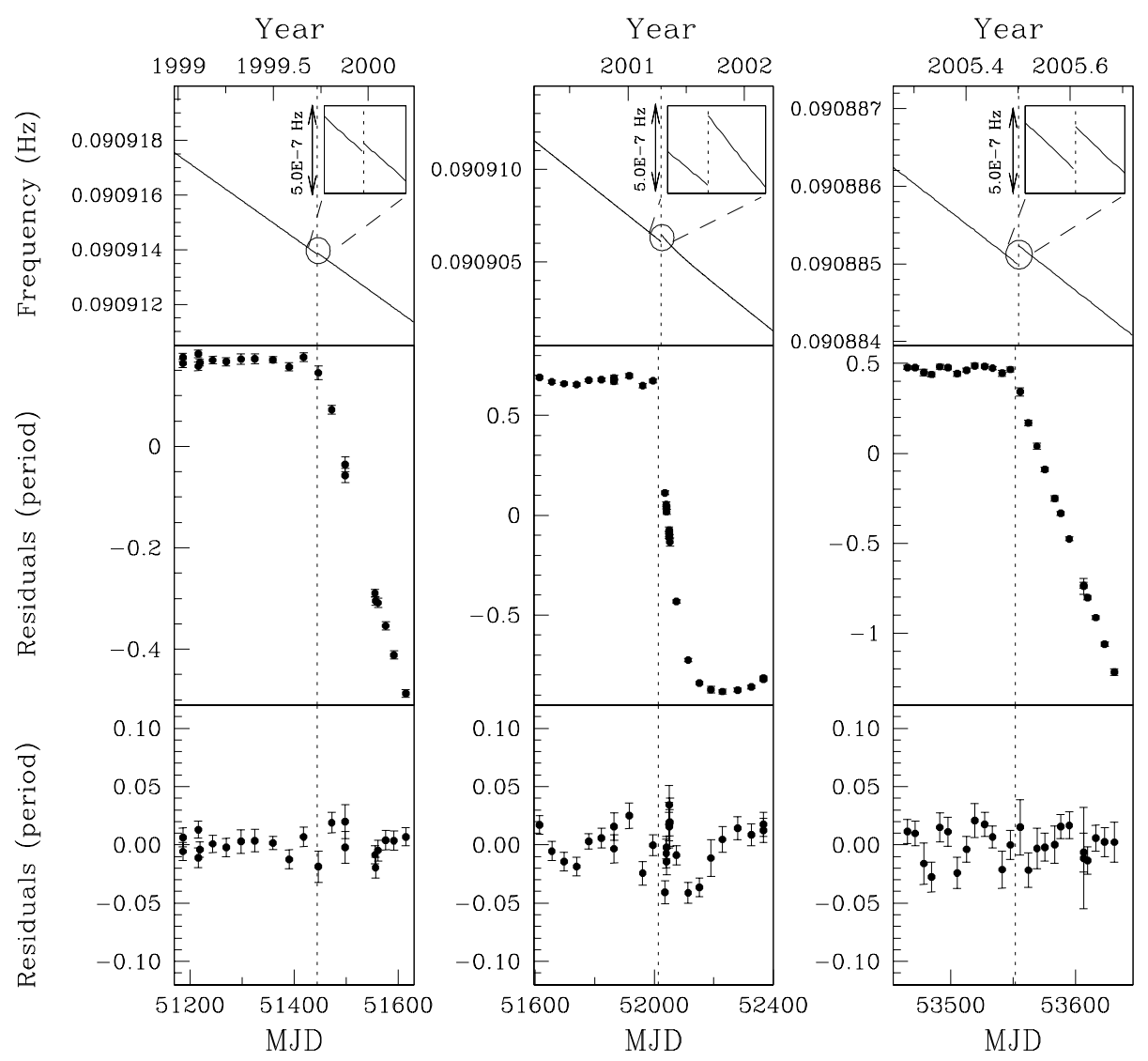

Fig. 4.- The three unambiguous glitches observed in RXS J170849.0-400910. Top panels: Frequency evolution around glitch as determined from ephemerides in Table 4, with the blow-up inset displaying glitch amplitude on a common scale for comparison. Middle panels: Residuals after subtraction of the best-fit pre-glitch ephemeris given in Table 4. Bottom panels: Residuals after subtraction of best-fit glitch models given in Table 4. All panels: Dashed vertical lines indicate assumed glitch epochs. 

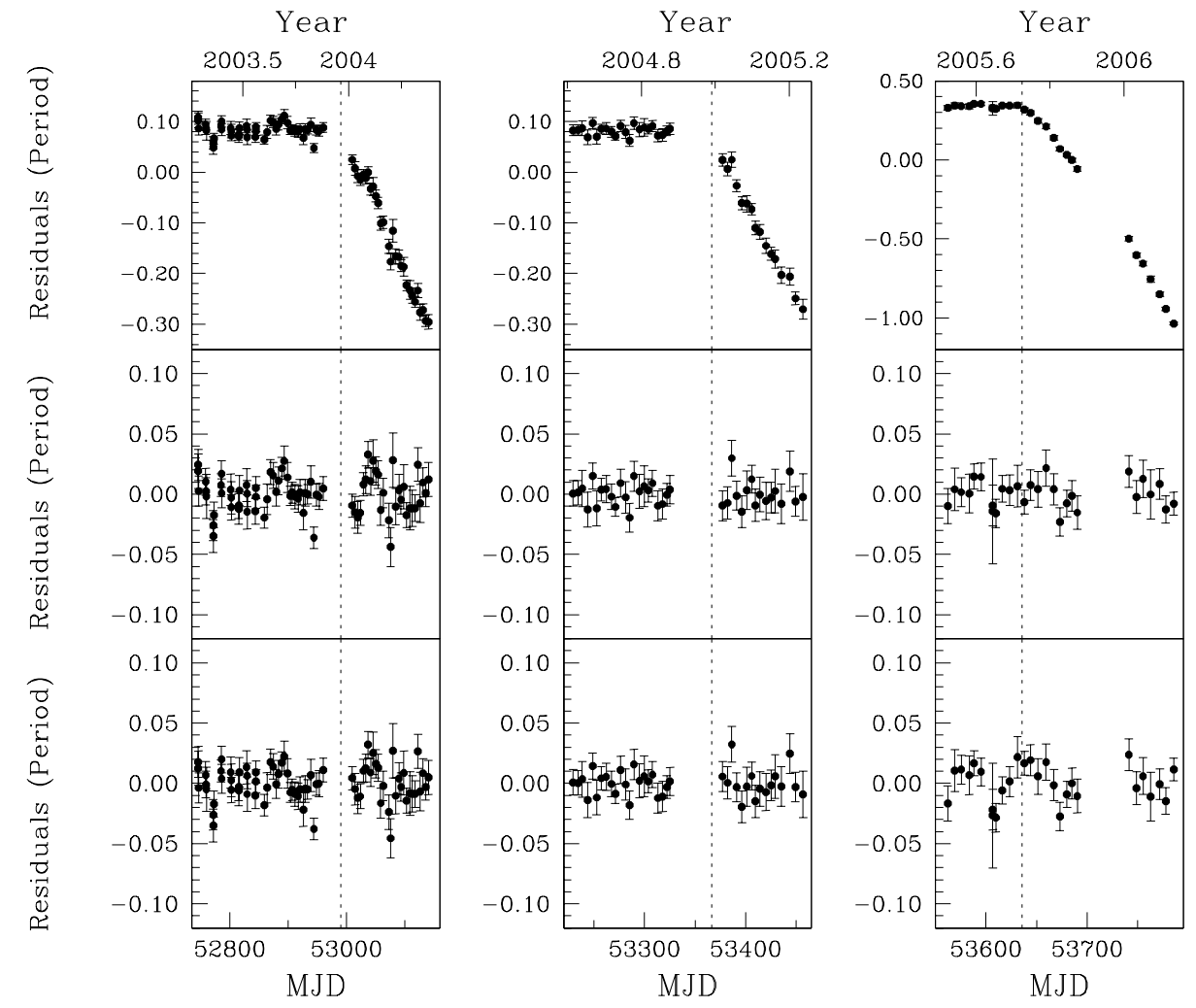

Fig. 5. - The three candidate glitches in RXS J170849.0-400910. Top panels: Residuals after subtraction of best-fit pre-'glitch' ephemeris given in Table 5. Middle panels: Residuals after subtraction of best-fit glitch models given in Table 5. Bottom panels: Residuals after subtraction of best-fit alternative models given in Table 6. All panels: Dashed vertical lines indicate assumed glitch epochs. 


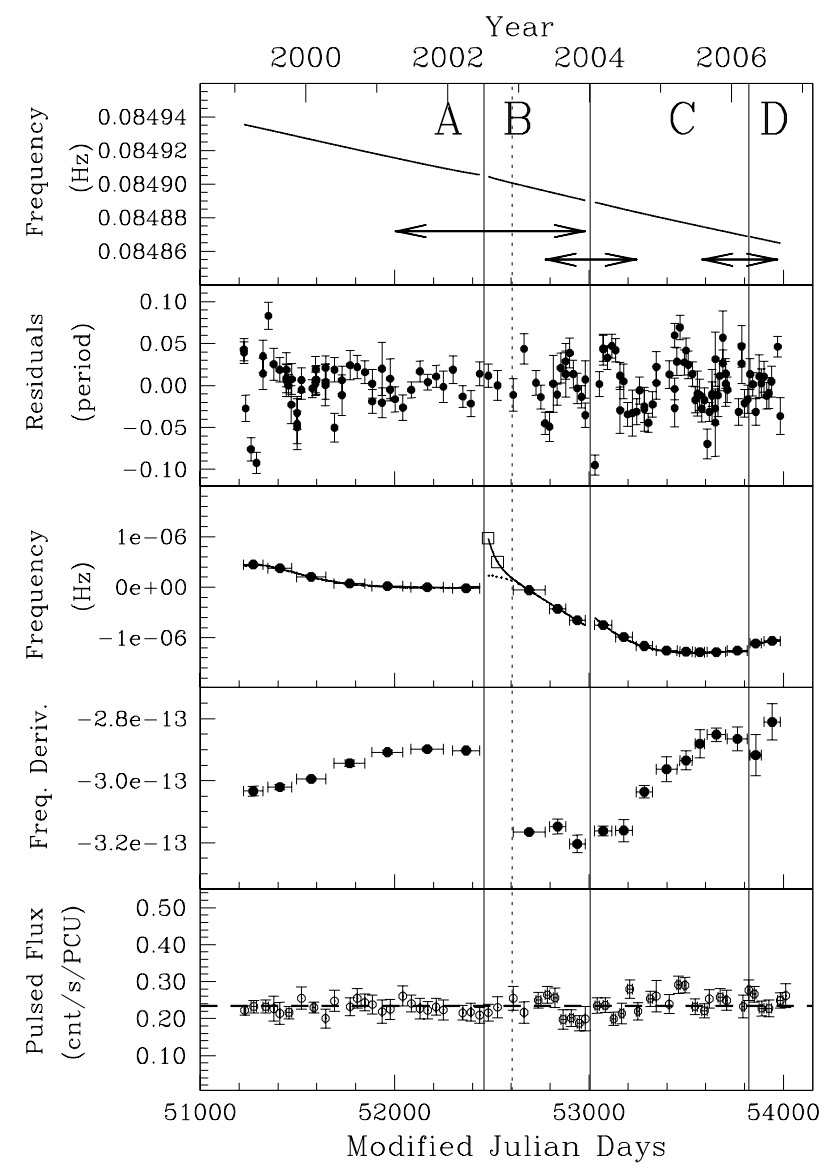

Fig. 6. - Spin and pulsed flux evolution in 1E 1841-045. Panels are described from top to bottom. Top: Frequency evolution, with inter-glitch intervals indicated for correspondence with ephemerides given in Table 7. Arrows indicate intervals for which glitch ephemerides were obtained (see Table 8). Next: Residuals, after subtraction of the best-fit models given in Table 7. Next: Solid curve: frequency evolution of the models shown in Table 7 after removal of the linear trend defined by the frequency and frequency derivative from the last year of data before the first glitch, as measured by fitting only those parameters. Dotted curve: alternate glitch recovery (see Section 3.2 for details). Filled circles: Measured frequencies in independent sub-intervals after subtraction of the extrapolation of the same linear trend. Unfilled squares: Epochs of the two immediate post-glitch observations (too few for the measurement of an independent frequency but crucial for the phase-coherent analysis). Next: Evolution of the frequency derivative in sub-intervals, when fitting locally for only $\nu$ and $\dot{\nu}$. Bottom: Pulsed flux in the $2-10 \mathrm{keV}$ range. All panels: Glitch epochs are indicated with solid vertical lines. The dashed vertical line indicates the start of ephemeris B2, which does not include the two immediate post-glitch observations (indicated with unfilled squares). 


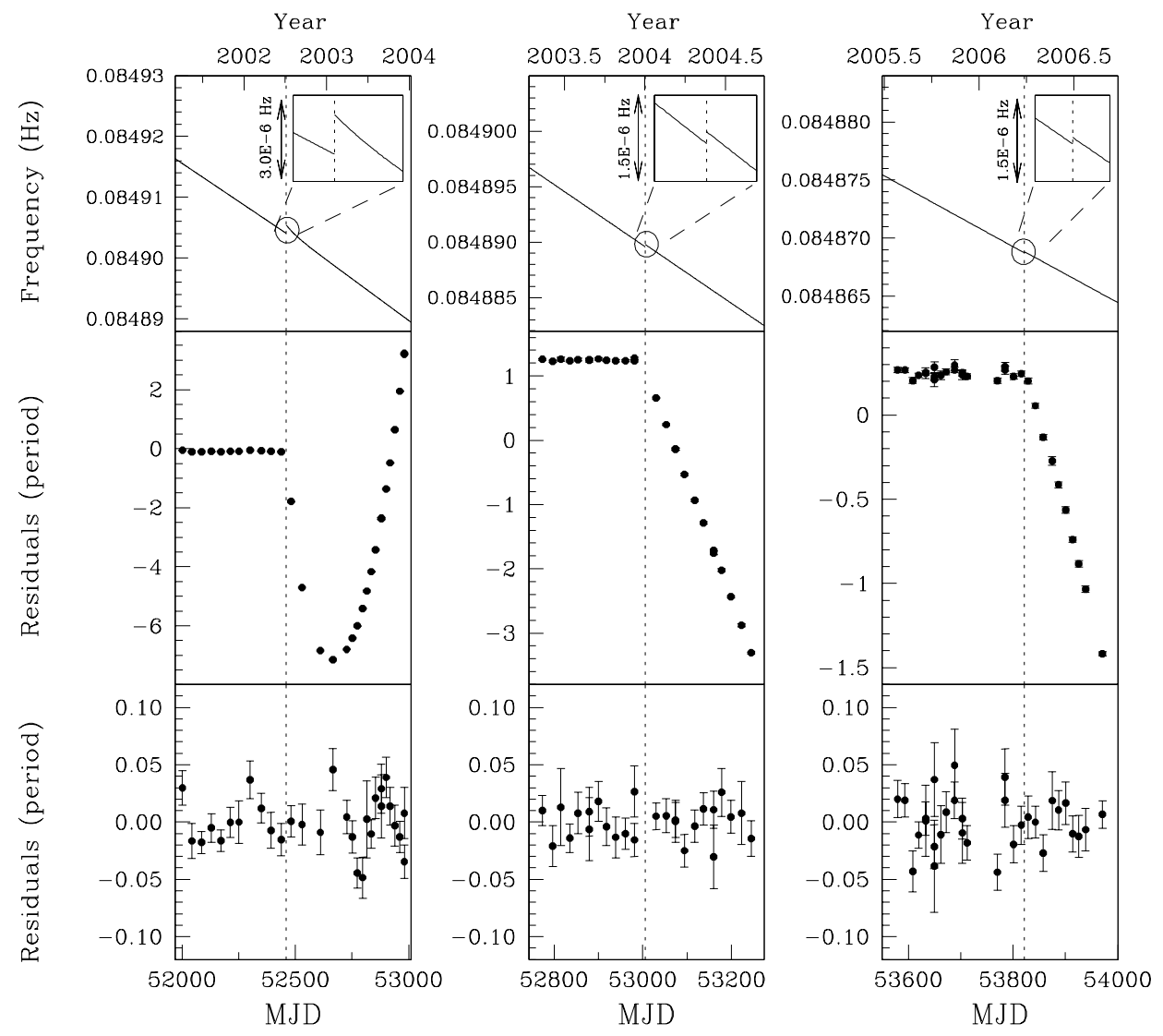

Fig. 7.- The three glitches in 1E 1841-045. Top panels: Frequency evolution around glitches as determined from ephemerides in Table 8, with the blow-up inset displaying glitch amplitude. Middle panels: Residuals after subtraction of the best-fit pre-glitch ephemeris given in Table 8. Bottom panels: Residuals after subtraction of best-fit glitch models given in Table 8, All panels: Dashed vertical lines indicate assumed glitch epochs. 


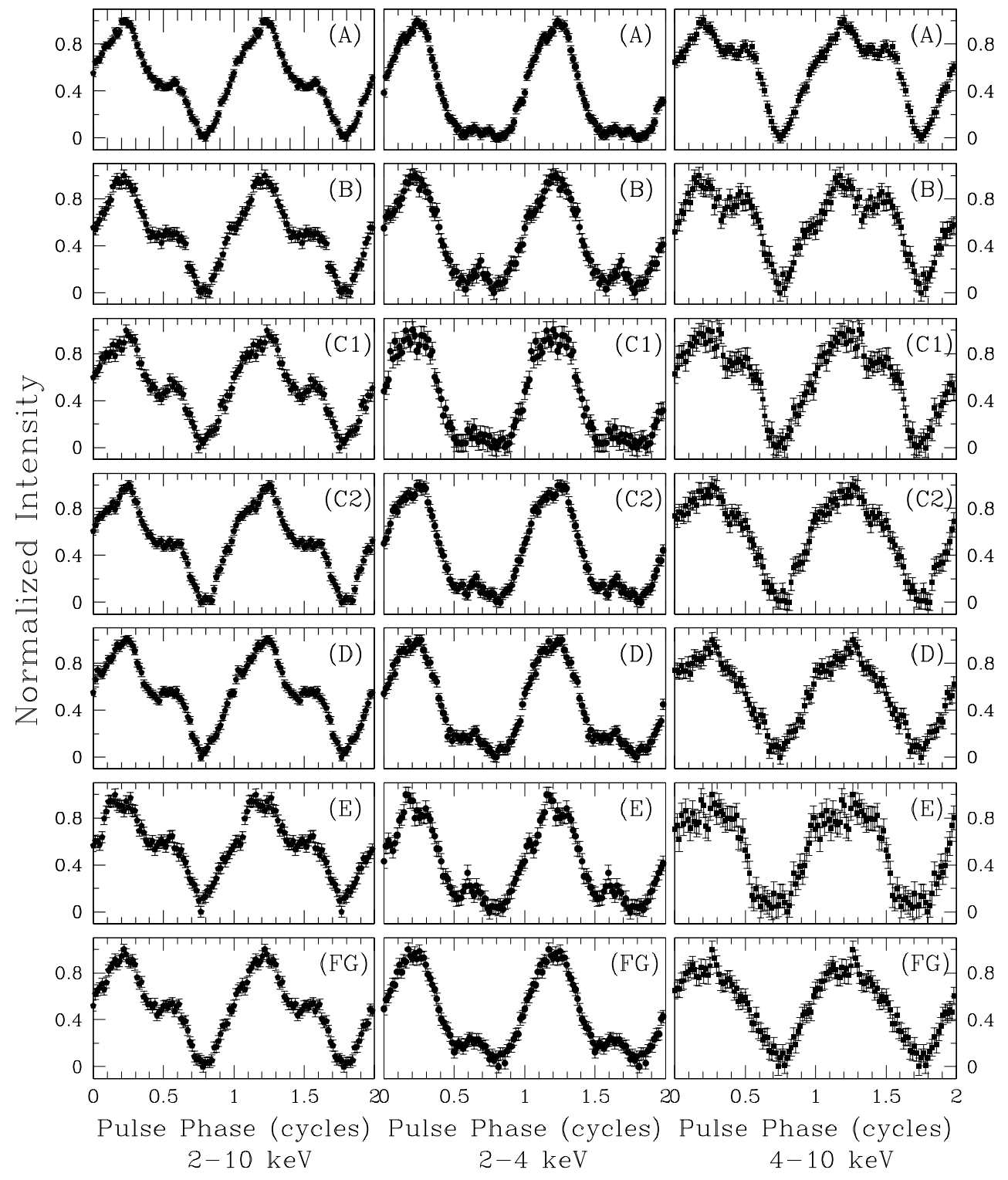

Fig. 8.- Normalized pulse profiles in three energy bands for RXS J170849.0-400910 for the seven glitch-free intervals (with corresponding labels at the top right) defined in the top panel of Figure 3. Different data qualities within an energy range are due to different net exposure times. Two cycles are shown for each profile for clarity. 


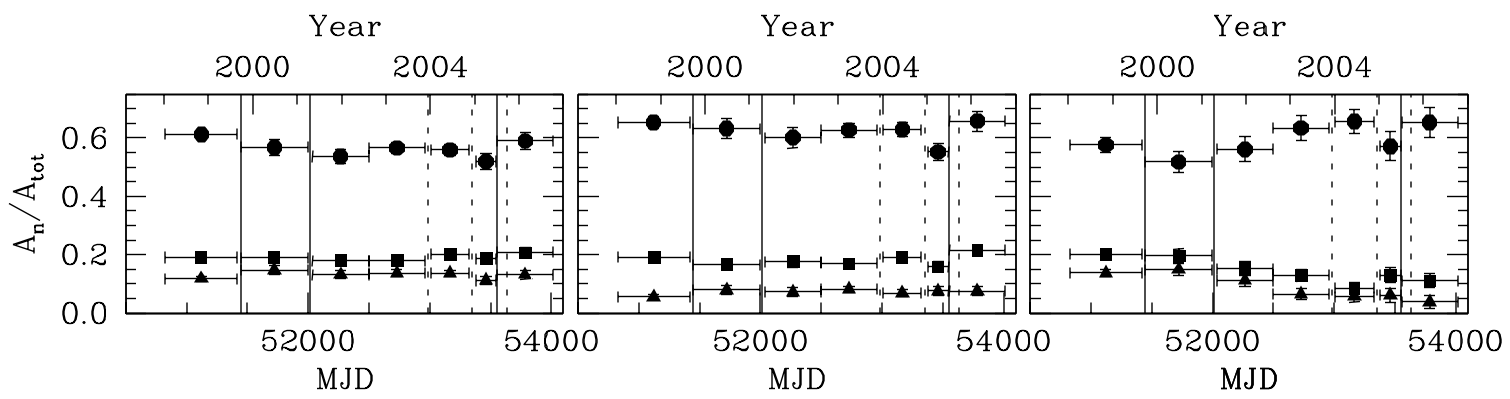

Fig. 9.- Left panel: Time evolution of the ratio of the power in the $n$th harmonic to the total power in the $2-10 \mathrm{keV}$ pulse profile of RXS J170849.0-400910. Circles represent $n=1$, squares $n=2$, and triangles $n=3$. Solid vertical lines indicate epochs of glitches; dashed vertical lines are epochs of candidate glitches. Middle panel: Same as left panel but for 2-4 keV. Right panel: Same as middle but for $4-10 \mathrm{keV}$. 


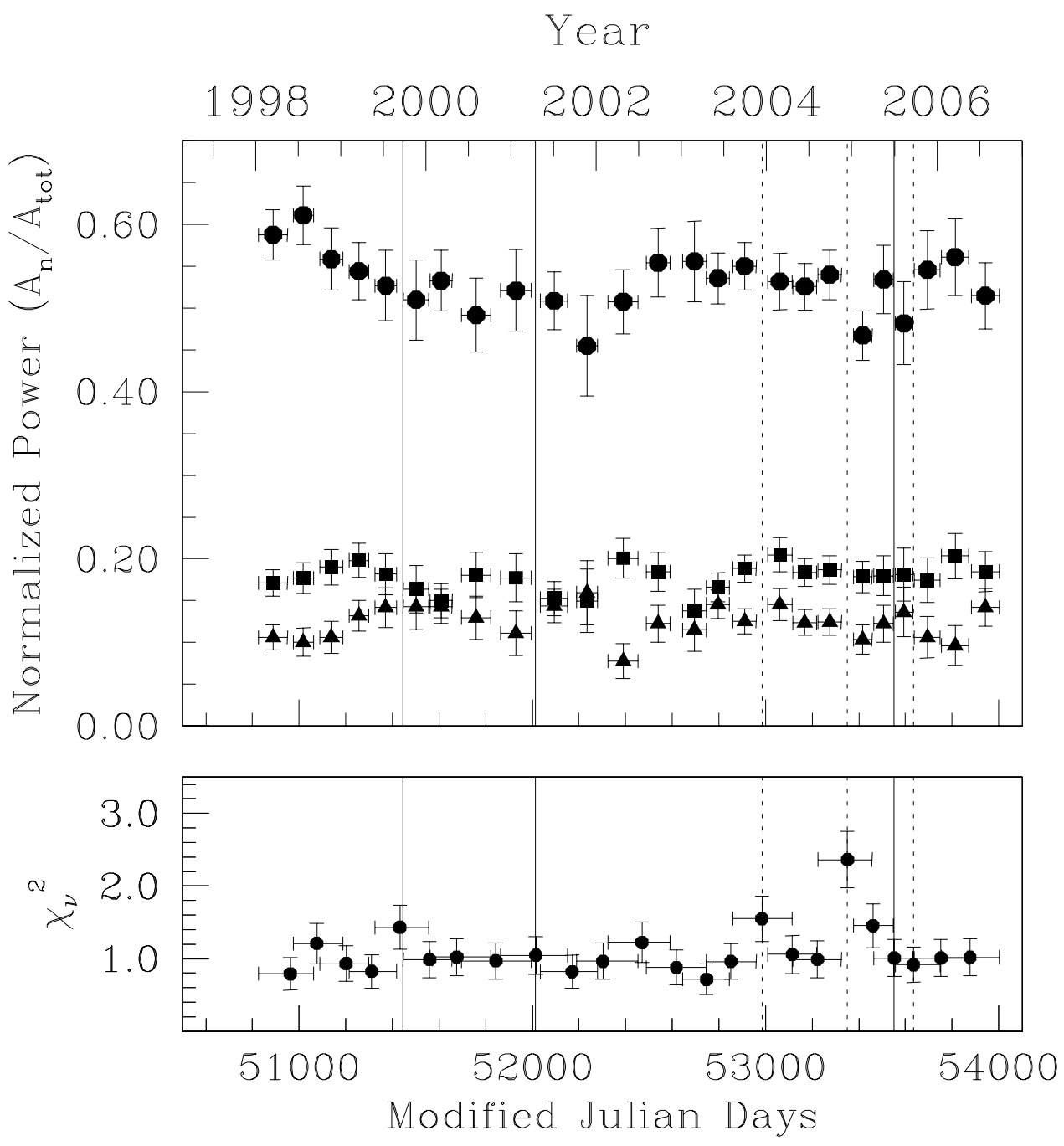

Fig. 10. - Top panel: Time evolution of the ratio of the power in the $n$th harmonic to the total power in the $2-10 \mathrm{keV}$ pulse profile of RXS J170849.0-400910. The circles represent $n=1$, squares $n=2$, and triangles $n=3$. Solid and dashed vertical lines indicate epochs of glitches and candidate glitches, respectively. The probability that the observed fluctuations are due to random noise are $68 \%, 97 \%$ and $69 \%$ for $n=1,2,3$, respectively. Bottom panel: reduced $\chi^{2}$ per degree of freedom for successive profile differences (see text $\$ 4.1$ for details). 


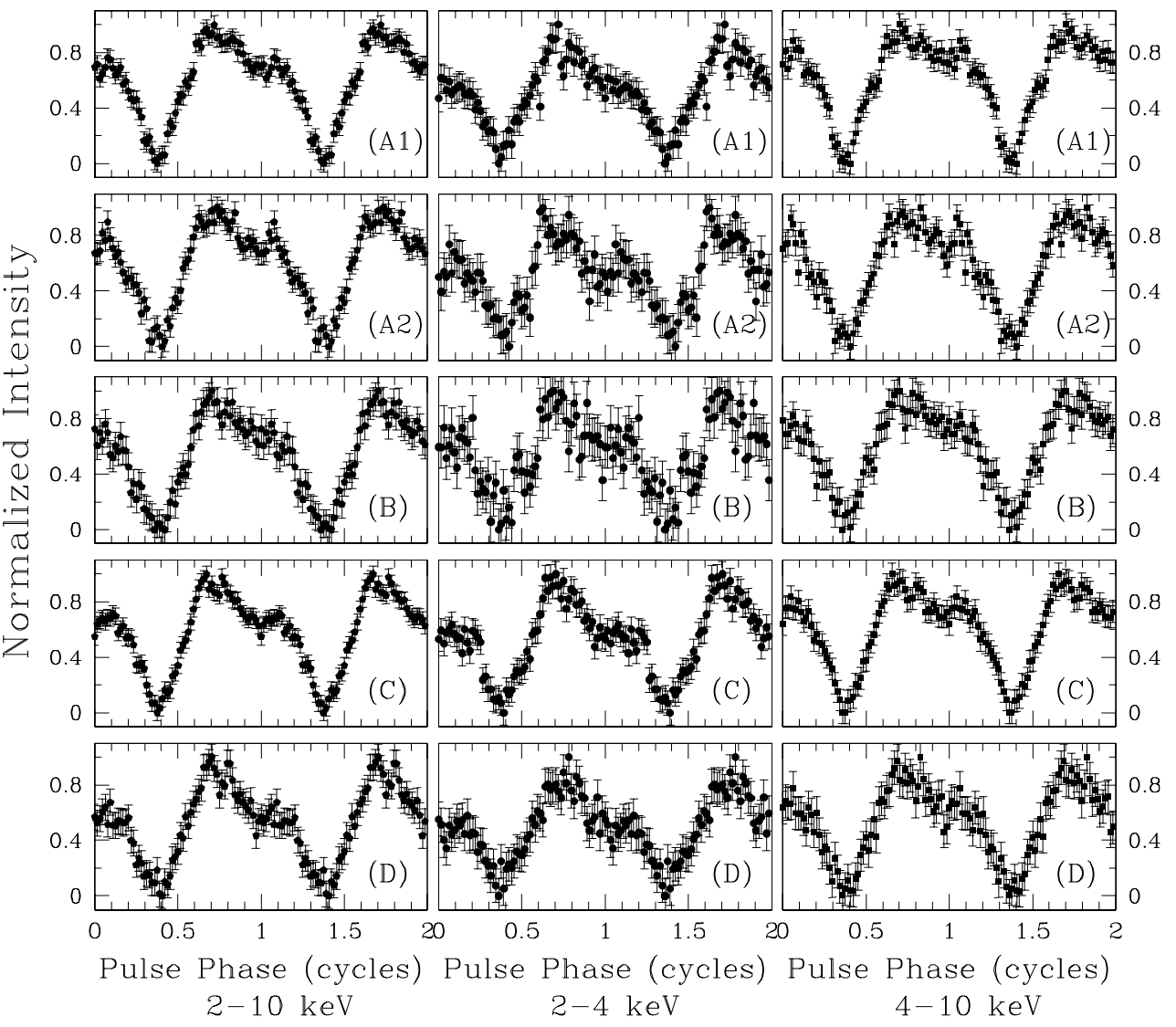

Fig. 11. - Normalized pulse profiles in three energy bands for 1E 1841-045 for the five glitch-free intervals defined in the top panel of Figure 6. Different data qualities in each energy range are due to different net exposure times. Two cycles are shown for each profile for clarity. 


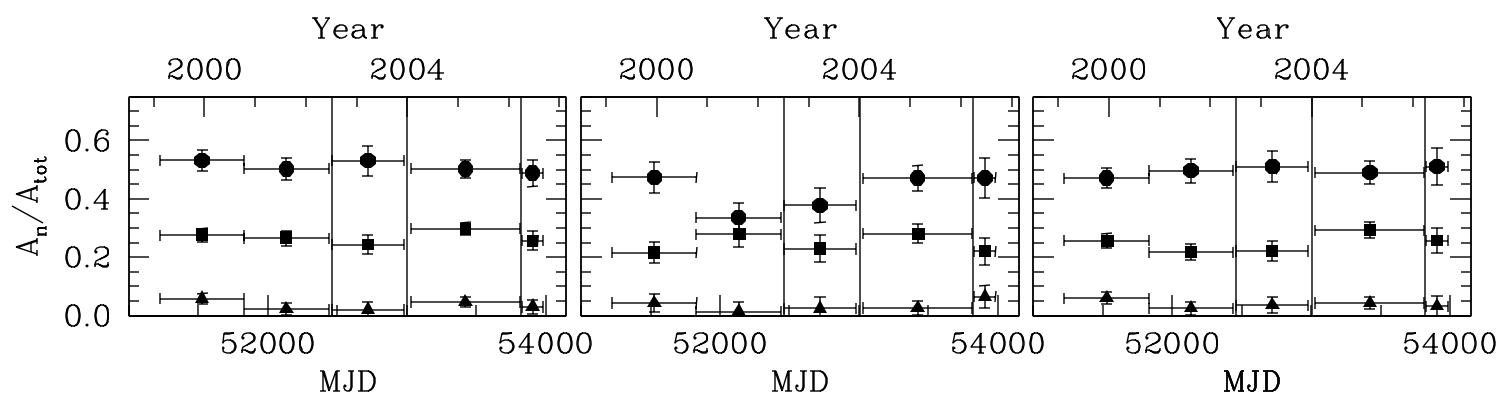

Fig. 12.- Left panel: Time evolution of the ratio of the power in the $n$th harmonic to the total power in the $2-10 \mathrm{keV}$ pulse profile of $1 \mathrm{E} 1841-045$. Circles represent $n=1$, squares $n=2$, and triangles $n=3$. Solid vertical lines indicate epochs of glitches. Middle panel: Same as left panel but for $2-4 \mathrm{keV}$. Right panel: Same as middle but for $4-10 \mathrm{keV}$. 

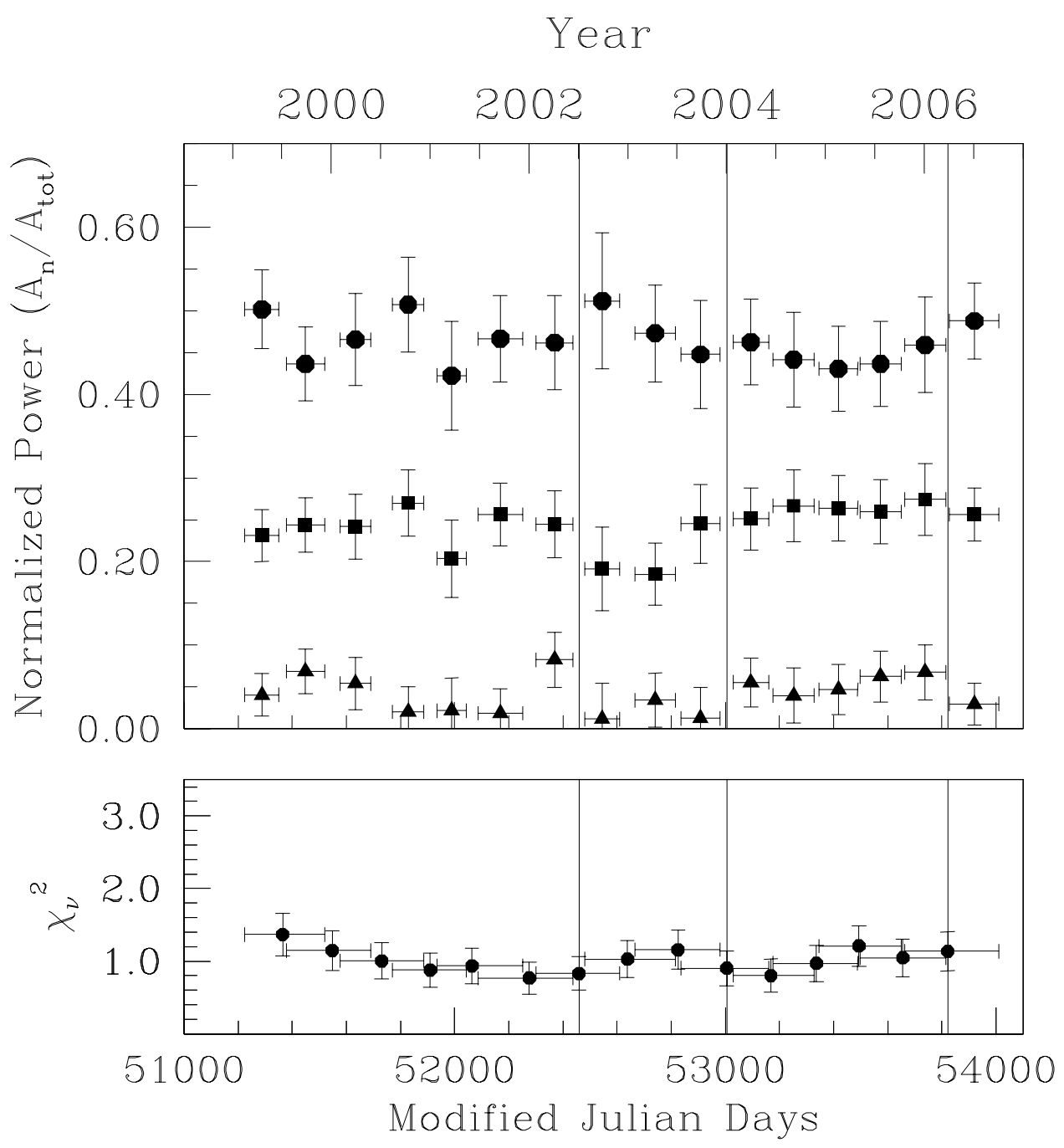

Fig. 13. - Top panel: Time evolution of the ratio of the power in the $n$th harmonic to the total power in the $2-10 \mathrm{keV}$ pulse profile of $1 \mathrm{E} 1841-045$. Circles represent $n=1$, squares $n=2$, and triangles $n=3$. Solid vertical lines indicate epochs of glitches. The probabilities that the observed fluctuations arise from random noise are 99\%, 97\% and $96 \%$ for $n=1,2,3$, respectively. Bottom panel: reduced $\chi^{2}$ per degree of freedom for successive profile differences (see text $\$ 4.2$ for details). 


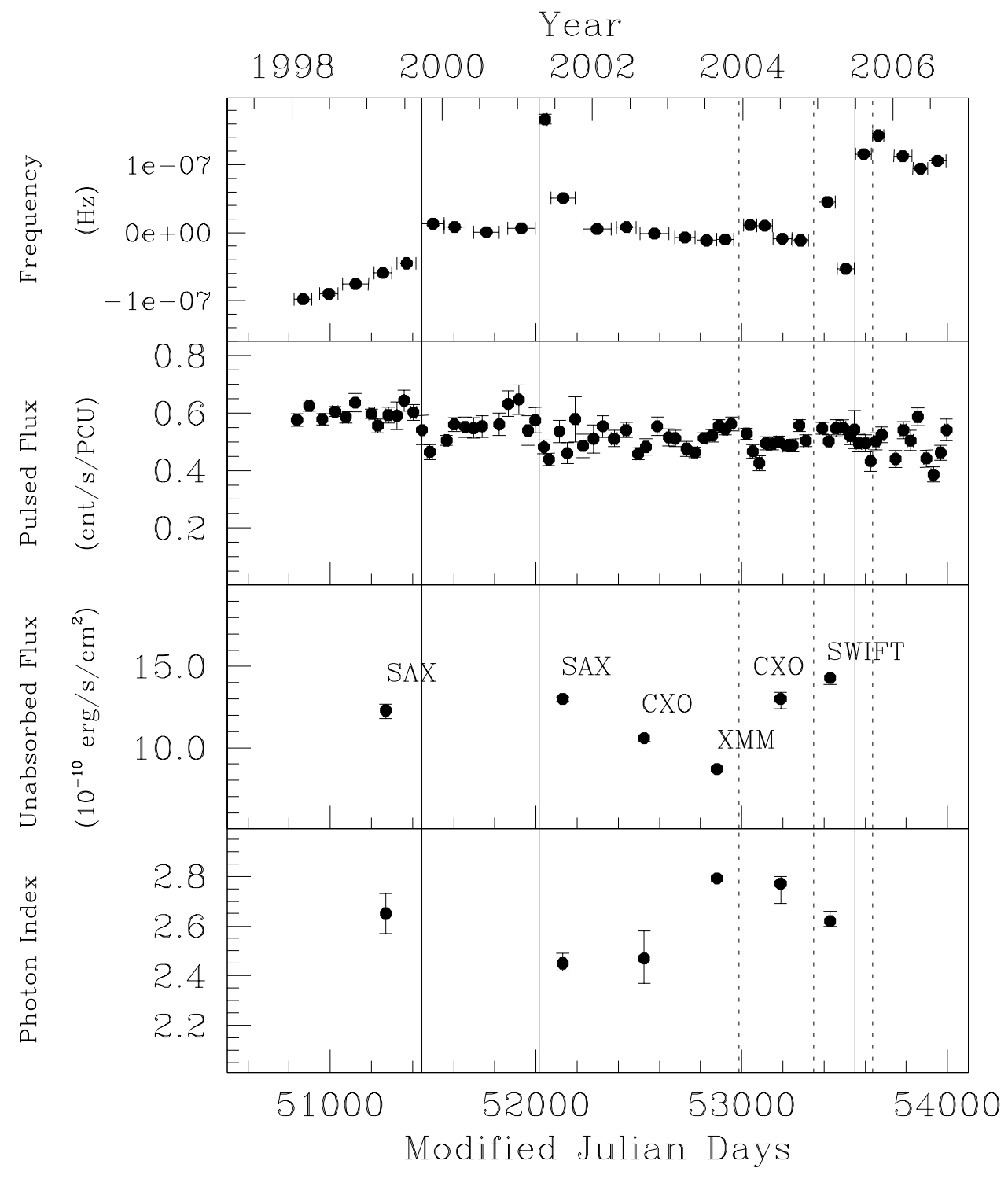

Fig. 14.- Frequency, pulsed flux, reported total unabsorbed flux, and reported photon index as a function of time for RXS J170849.0-400910. Frequency and pulsed flux data are identical to those shown in Figure 3, Solid and dashed vertical lines indicate epochs of glitches and glitch candidates, respectively. Unabsorbed phase-averaged $0.5-10 \mathrm{keV}$ fluxes and photon indexes are from Rea et al. (2005) and Campana et al. (2007), and are labelled by observing telescope. That the pulsed flux remains relatively constant while the phaseaveraged flux appears to vary by nearly a factor of two (albeit as measured by different instruments) suggests a strong anti-correlation between total flux and pulsed fraction. 

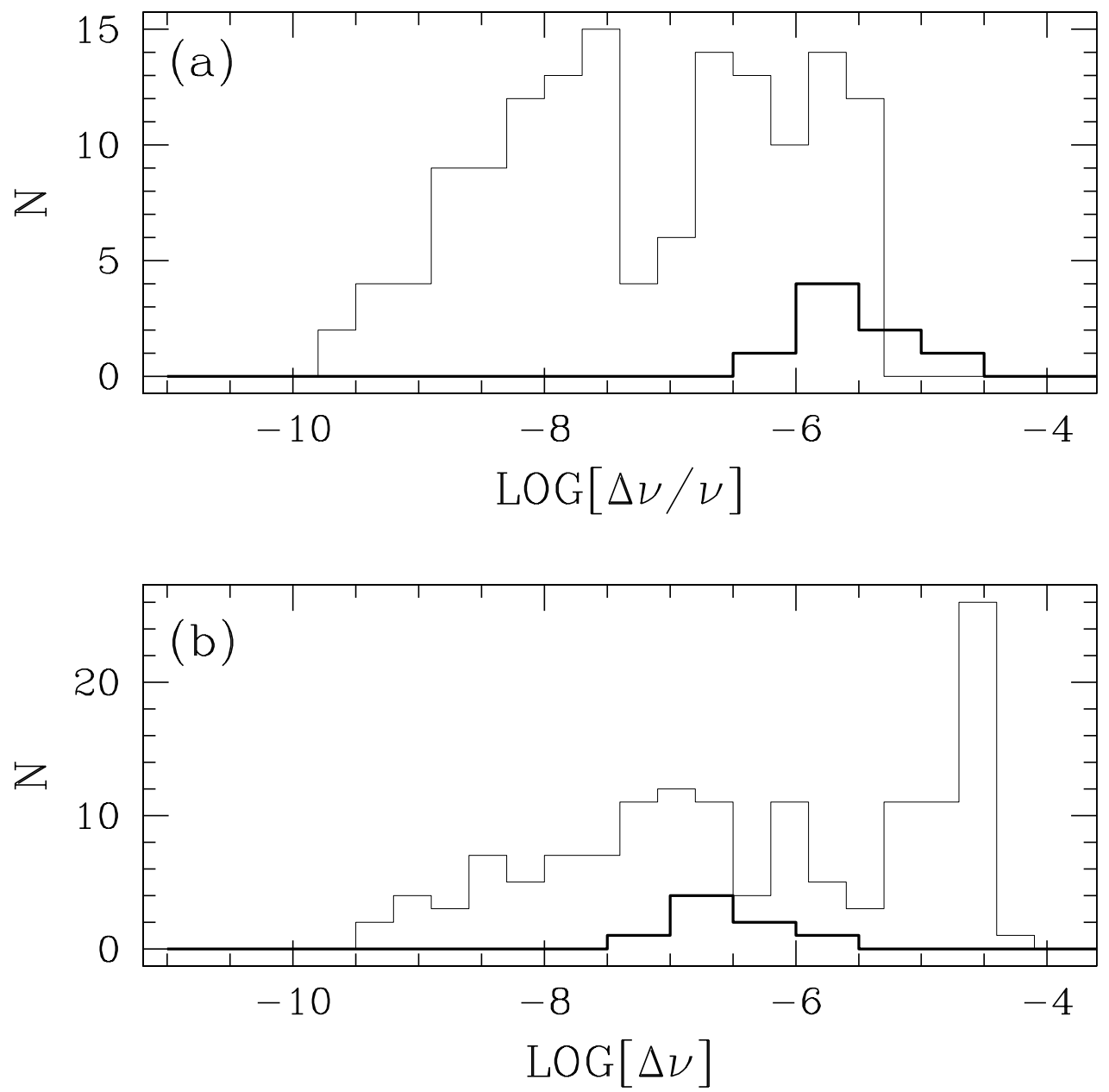

Fig. 15.- Amplitude distribution of AXP glitches (bold line) and radio pulsar glitches (thin line) for (a) fractional frequency jump and (b) absolute frequency jump (in $\mathrm{Hz}$ ). Radio pulsar glitch amplitudes are from an unpublished catalog kindly supplied by A. Lyne. AXP glitches included here are those listed in Tables 4 and 8, the 2002 1E 2259+586 glitch (Kaspi et al. 2003; Woods et al. 2004), as well as a recent unpublished 1E 2259+586 glitch (Dib et al., in prep.). 

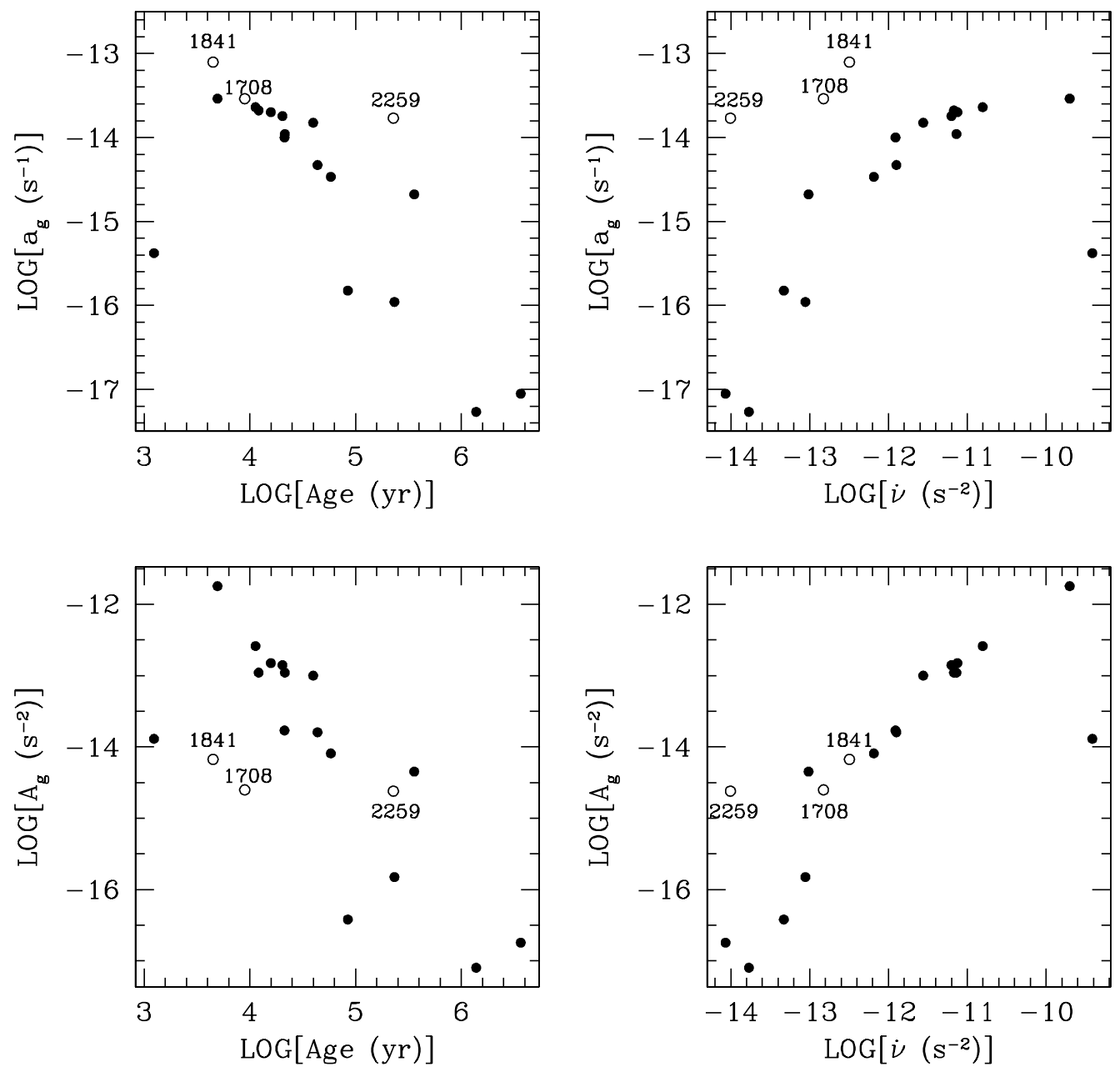

Fig. 16.- Activity parameters versus age (as estimated from $\nu / 2 \dot{\nu}$ ) and versus $\dot{\nu}$ for radio pulsars and AXPs. Fractional activity $a_{g}$ is defined using the sum of the fractional frequency changes, while activity $A_{g}$ is defined using the absolute frequency jumps. The only radio pulsars included (filled circles) are those having exhibited three glitches or more during continual (e.g., bi-monthly) monitoring, as recorded in the unpublished glitch catalog kindly supplied by A. Lyne. The AXPs included here (open circles) are RXS J170849.0-400910, 1E 1841-045, and 1E 2259+586. The latter has glitched twice, once in 2002 (Kaspi et al. 2003; Woods et al. 2004), and once in 2007 (Dib et al., in prep.). Only unambiguous glitches were included for RXS J170849.0-400910; as the candidate glitches are small, including them does not make a qualitative difference. 


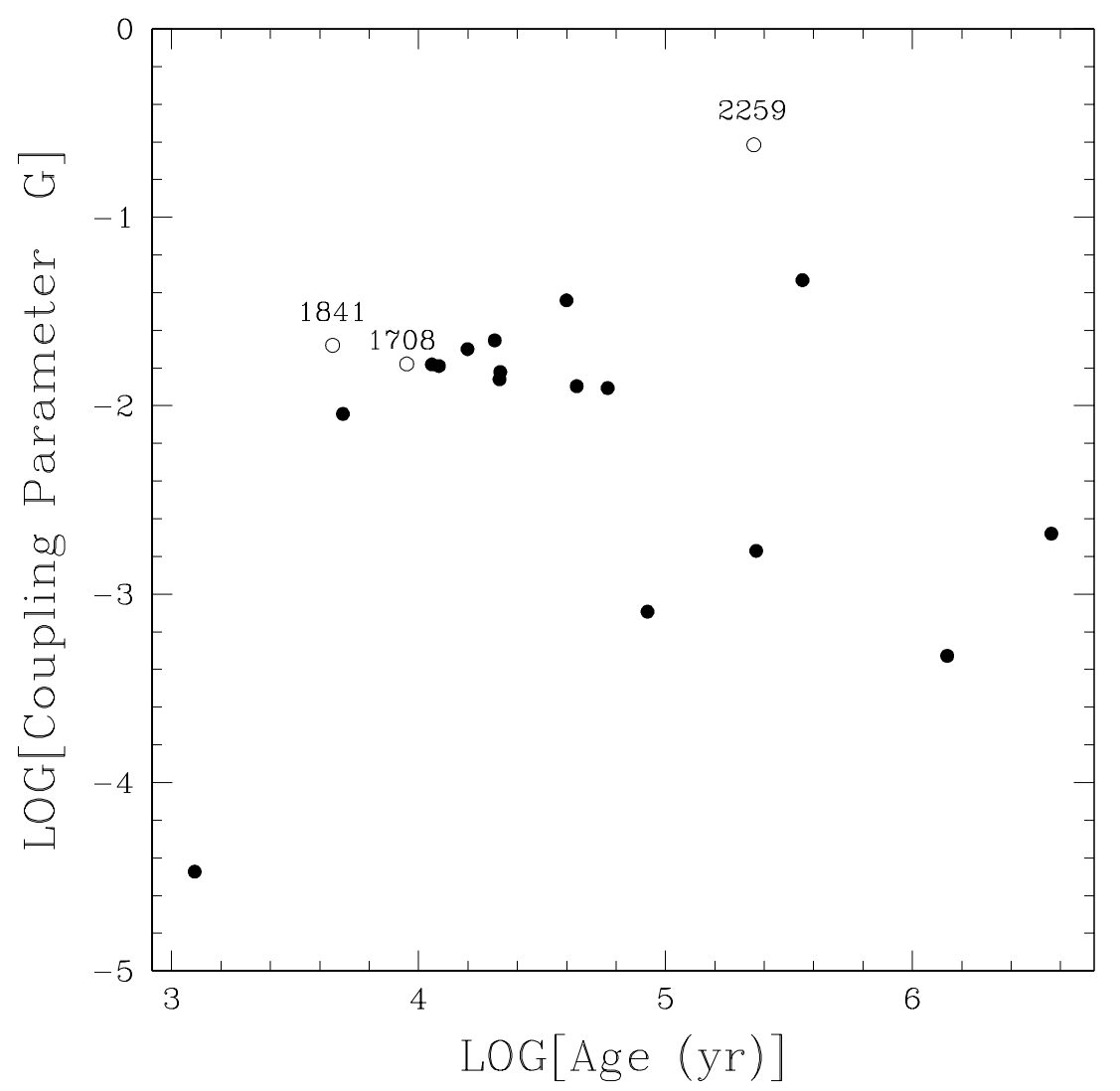

Fig. 17.- "Coupling parameter" $G$ (as defined by (Link et al. 1999); see $\$ 6.1$ ) as a function of spin-down age $(\nu / 2 \dot{\nu})$. Solid points are radio pulsars with three or more observed glitches, as recorded in the unpublished catalog of A. Lyne. Open circles are AXPs 1E 2259+586, RXS J170849.0-400910, and 1E 1841-045. 
Table 1. Summary of RXTE Observations of RXS J170849.0-400910

\begin{tabular}{ccccccc}
\hline \hline $\begin{array}{c}\text { Obs. } \\
\text { Cycle }\end{array}$ & $\begin{array}{c}\text { Typical } \\
\text { Exp. }{ }^{2} \\
(\mathrm{ks})\end{array}$ & $\begin{array}{c}\text { Typical } \\
\text { Separation }^{\mathrm{a}} \\
\text { (days) }\end{array}$ & $\begin{array}{c}\text { No. of } \\
\text { Obs. }^{{ }^{3}}\end{array}$ & $\begin{array}{c}\text { Total } \\
\text { Exp. }^{\mathrm{c}} \\
(\mathrm{ks})\end{array}$ & $\begin{array}{c}\text { First }- \text { Last } \\
\text { MJD }^{\mathrm{d}}\end{array}$ & First Date - Last Date \\
\hline 3 & 2.5 & 15 & 29 & 75 & $50825.7-51186.7$ & $01 / 12 / 1998-01 / 08 / 1999$ \\
4 & 3 & 24 & 20 & 60 & $51215.7-51614.1$ & $02 / 06 / 1999-03 / 11 / 2000$ \\
5 & 3 & 24 & 13 & 40 & $51655.7-52041.5$ & $04 / 21 / 2000-05 / 12 / 2001$ \\
6 & 3 & 29 & 13 & 40 & $52049.5-52325.6$ & $05 / 20 / 2001-02 / 20 / 2002$ \\
7 & 5.5 & 23 & 12 & 65 & $52366.5-52718.7$ & $04 / 02 / 2002-03 / 20 / 2003$ \\
8 & 1.8 & 5 & 58 & 105 & $52745.7-53058.6$ & $04 / 16 / 2003-02 / 23 / 2004$ \\
9 & 2 & 5 & 70 & 135 & $53063.1-53429.1$ & $02 / 28 / 2004-02 / 28 / 2005$ \\
10 & 2 & 8 & 47 & 90 & $53435.1-53791.6$ & $03 / 06 / 2005-02 / 25 / 2006$ \\
$11^{\mathrm{e}}$ & 2 & 7 & 32 & 60 & $53799.0-54015.4$ & $03 / 05 / 2006-10 / 07 / 2006$ \\
\hline
\end{tabular}

${ }^{a}$ The exposure and separation are approximate. Note that the PCA effective area changed with time primarily due the reduction of the average number of PCUs operational during an integration. This effect is not incorporated in the tabulated integration times.

${ }^{\mathrm{b}}$ When the last digits of the observation ID of two successive data sets are different, the two data sets are considered separate observations.

${ }^{c}$ The total exposure does not include Earth occultation periods.

${ }^{\mathrm{d}}$ First MJD and Last MJD are the epochs, in Modified Julian Days, of the first and the last observations in a Cycle.

${ }^{\mathrm{e}}$ Cycle 11 not yet completed. 
Table 2. Summary of RXTE Observations of 1E 1841-045

\begin{tabular}{ccccccc}
\hline \hline $\begin{array}{c}\text { Obs. } \\
\text { Cycle }\end{array}$ & $\begin{array}{c}\text { Typical } \\
\text { Exp. }^{\mathrm{a}} \\
(\mathrm{ks})\end{array}$ & $\begin{array}{c}\text { Typical } \\
\text { Separation }^{\mathrm{a}} \\
\text { (days) }\end{array}$ & $\begin{array}{c}\text { No. of } \\
\text { Obs. }^{\mathrm{b}}\end{array}$ & $\begin{array}{c}\text { Total } \\
\text { Exp. }^{\mathrm{c}} \\
(\mathrm{ks})\end{array}$ & $\begin{array}{c}\text { First }- \text { Last } \\
\text { MJD }^{\mathrm{d}}\end{array}$ & First Date - Last Date \\
\hline 4 & 4.5 & 27 & 26 & 120 & $51224.4-51597.3$ & $02 / 15 / 1999-02 / 23 / 2000$ \\
5 & 4.5 & 38 & 16 & 70 & $51644.7-51976.9$ & $04 / 10 / 2000-03 / 08 / 2001$ \\
6 & 7 & 27 & 8 & 50 & $52001.6-52300.1$ & $04 / 02 / 2001-01 / 26 / 2002$ \\
7 & 12 & 45 & 7 & 80 & $52349.8-52666.0$ & $03 / 16 / 2002-01 / 27 / 2003$ \\
8 & 4.5 & 20 & 17 & 80 & $52726.8-53052.9$ & $03 / 28 / 2003-02 / 17 / 2004$ \\
9 & 4.5 & 20 & 19 & 80 & $53073.7-53413.3$ & $03 / 09 / 2004-02 / 12 / 2005$ \\
10 & 5 & 14 & 31 & 130 & $53440.0-54153.9$ & $03 / 11 / 2005-02 / 22 / 2007$ \\
$11^{\mathrm{e}}$ & 5 & 14 & 12 & 60 & $53800.9-53970.6$ & $03 / 06 / 2006-08 / 23 / 2006$ \\
\hline
\end{tabular}

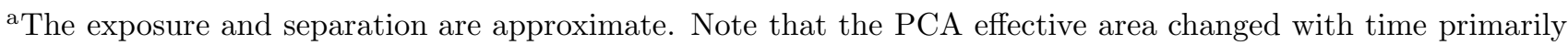
due the reduction of the average number of PCUs operational during an integration. This effect is not incorporated in the tabulated integration times.

${ }^{b}$ When the last digits of the observation ID of two successive data sets are different, the two data sets are considered separate observations.

${ }^{\mathrm{c}}$ The total exposure does not include Earth occultation periods.

${ }^{\mathrm{d}}$ First MJD and Last MJD are the epochs, in Modified Julian Days, of the first and the last observations in a Cycle.

${ }^{\mathrm{e}}$ Cycle 11 not yet completed. 
Table 3. Long-Term Spin Parameters for RXS J170849.0-400910a

\begin{tabular}{|c|c|c|c|c|c|c|c|}
\hline Parameter & $\begin{array}{c}\text { Ephemeris A } \\
\text { Spanning MJD } \\
50826-51418\end{array}$ & $\begin{array}{c}\text { Ephemeris B } \\
\text { Spanning MJD } \\
51446-51996\end{array}$ & $\begin{array}{c}\text { Ephemeris C } \\
\text { Spanning MJD } \\
52036-52960\end{array}$ & $\begin{array}{c}\text { Ephemeris D } \\
\text { Spanning MJD } \\
53010-53325\end{array}$ & $\begin{array}{c}\text { Ephemeris E } \\
\text { Spanning MJD } \\
53377-53548\end{array}$ & $\begin{array}{c}\text { Ephemeris F } \\
\text { Spanning MJD } \\
53556-53631\end{array}$ & $\begin{array}{c}\text { Ephemeris G } \\
\text { Spanning MJD } \\
53638-54015\end{array}$ \\
\hline MJD start & 50826.078 & 51446.610 & 52035.655 & 53010.094 & 53377.133 & 53555.734 & 53638.033 \\
\hline MJD end & 51418.374 & 51995.680 & 52960.186 & 53325.061 & 53547.811 & 53631.161 & 54015.487 \\
\hline TOAs & 39 & 19 & 74 & 69 & 29 & 13 & 49 \\
\hline$\nu(\mathrm{Hz})$ & $0.090913818(2)$ & $0.090906071(3)$ & $0.090906089(3)$ & $0.090892731(13)$ & $0.090887608(18)$ & $0.090885281(8)$ & $0.090884082(9)$ \\
\hline$\dot{\nu}\left(10^{-13} \mathrm{~Hz} \mathrm{~s}^{-1}\right)$ & $-1.583(3)$ & $-1.574(2)$ & $-1.565(6)$ & $-1.40(5)$ & $-1.19(8)$ & $-1.70(2)$ & $-1.58(3)$ \\
\hline$\ddot{\nu}\left(10^{-22} \mathrm{~Hz} \mathrm{~s}^{-2}\right)$ & $-1.4(3)$ & $0.36(9)$ & $-8.9(1.8)$ & $-44(11)$ & $-131(23)$ & - & $-8(7)$ \\
\hline$d^{3} \nu / d t^{3}\left(10^{-28} \mathrm{~Hz} \mathrm{~s}^{-3}\right)$ & $-0.056(9)$ & - & $1.5(3)$ & $5.5(1.6)$ & $15(3)$ & - & $1.4(9)$ \\
\hline$d^{4} \nu / d t^{4}\left(10^{-35} \mathrm{~Hz} \mathrm{~s}^{-4}\right)$ & - & - & $-1.4(3)$ & $-3.1(1.0)$ & - & - & $-0.8(6)$ \\
\hline$d^{5} \nu / d t^{5}\left(10^{-43} \mathrm{~Hz} \mathrm{~s}^{-5}\right)$ & - & - & $7.4(1.5)$ & - & - & - & - \\
\hline$d^{6} \nu / d t^{6}\left(10^{-50} \mathrm{~Hz} \mathrm{~s}^{-6}\right)$ & - & - & $-1.8(4)$ & - & - & - & - \\
\hline$\Delta \nu_{d}^{\mathrm{b}}(\mathrm{Hz})$ & - & - & $36(3) \times 10^{-08}$ & - & - & - & - \\
\hline$t_{d} \mathrm{~b}^{\mathrm{a}}$ (days) & - & - & $43(2)$ & - & - & - & - \\
\hline Epoch (MJD) & 51445.3846 & 52016.48413 & 52016.48413 & 52989.8475 & 53366.3150 & 53549.15095 & 53635.6772 \\
\hline RMS residual (phase) & 0.0079 & 0.0150 & 0.0154 & 0.0132 & 0.0142 & 0.0112 & 0.0154 \\
\hline
\end{tabular}

a Numbers in parentheses are TEMPO-reported $1 \sigma$ uncertainties.

${ }^{\mathrm{b}}$ Parameters held fixed at values determined from local glitch fits as shown in Table 4 
Table 4. Local Ephemerides of RXS J170849.0-400910 Near Glitch Epochs ${ }^{\mathrm{a}}$

\begin{tabular}{lccc}
\hline \hline \multicolumn{1}{c}{ Parameter } & Ephemeris & Ephemeris & Ephemeris \\
& Near Glitch 1 & Near Glitch 2 & Near Glitch 3 \\
\hline MJD range & $51186.503-51614.187$ & $51614.185-52366.663$ & $53465.392-53631.161$ \\
TOAs & 22 & 29 & 26 \\
Epoch (MJD) & 51445.3846 & 52016.48413 & 53549.15095 \\
$\nu(\mathrm{Hz})$ & $0.090913822(2)$ & $0.090906068(2)$ & $0.090885035(9)$ \\
$\dot{\nu}\left(10^{-13} \mathrm{~Hz} \mathrm{~s}^{-1}\right)$ & $-1.5714(14)$ & $-1.5797(11)$ & $-1.67(2)$ \\
Glitch Epoch $(\mathrm{MJD})$ & 51445.3846 & 52016.48413 & 53549.15095 \\
$\Delta \nu(\mathrm{Hz})$ & $5.1(3) \times 10^{-8}$ & $2.2(4) \times 10^{-8}$ & $24.6(9) \times 10^{-8}$ \\
$\Delta \dot{\nu}\left(\mathrm{Hz} \mathrm{s}{ }^{-1}\right)$ & $-0.8(4) \times 10^{-15}$ & $-1.1(2) \times 10^{-15}$ & $-2(2) \times 10^{-15}$ \\
$\Delta \nu_{d}(\mathrm{~Hz})$ & - & $36(3) \times 10^{-8}$ & - \\
$t_{d}($ days $)$ & - & $43(2)$ & - \\
RMS residual (phase) & 0.0102 & 0.0193 & 0.0140 \\
\hline
\end{tabular}

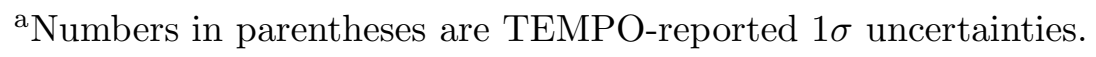


Table 5. Local Ephemerides of RXS J170849.0-400910 Near Candidate Glitch Epochs $^{\mathrm{a}}$

\begin{tabular}{lccc}
\hline \hline \multicolumn{1}{c}{ Parameter } & $\begin{array}{c}\text { Ephemeris } \\
\text { Near Candidate } 1\end{array}$ & $\begin{array}{c}\text { Ephemeris } \\
\text { Near Candidate 2 }\end{array}$ & $\begin{array}{c}\text { Ephemeris } \\
\text { Near Candidate 3 }\end{array}$ \\
\hline MJD range & $52745.790-53140.604$ & $53229.271-53456.688$ & $53562.209-53785.652$ \\
TOAs & 78 & 38 & 28 \\
Epoch (MJD) & 529898475 & 53366.3150 & 53635.6772 \\
$\nu(\mathrm{Hz})$ & $0.0908927493(18)$ & $0.090887617(5)$ & $0.090884020(8)$ \\
$\dot{\nu}\left(10^{-13} \mathrm{~Hz} \mathrm{~s}^{-1}\right)$ & $-1.5842(15)$ & $-1.570(7)$ & $-1.67(2)$ \\
Glitch Epoch $(\mathrm{MJD})$ & 529898475 & 53366.3150 & 53635.6772 \\
$\Delta \nu(\mathrm{Hz})$ & $2.8(4) \times 10^{-8}$ & $5.2(6) \times 10^{-8}$ & $6.7(3) \times 10^{-8}$ \\
$\Delta \dot{\nu}\left(\mathrm{Hz} \mathrm{s}{ }^{-1}\right)$ & $0^{\mathrm{b}}$ & $-1.9(1.3) \times 10^{-15}$ & $6.0(5) \times 10^{-15}$ \\
$\Delta \nu_{d}(\mathrm{~Hz})$ & - & - & - \\
$t_{d}($ days $)$ & - & - & - \\
RMS residual (phase) & 0.0153 & 0.0099 & 0.0110 \\
\hline
\end{tabular}

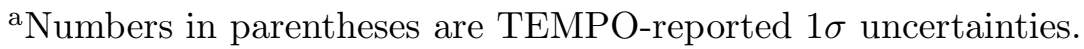

${ }^{\mathrm{b}}$ Entries with the value ' 0 ' are consistent with being zero. 
Table 6. Alternate Ephemerides of RXS J170849.0-400910 Near Candidate Glitch Epochs ${ }^{\mathrm{a}}$

\begin{tabular}{lccc}
\hline \hline & $\begin{array}{c}\text { Ephemeris } \\
\text { Parameter }\end{array}$ & $\begin{array}{c}\text { Ephemeris } \\
\text { Near Candidate 2 }\end{array}$ & $\begin{array}{c}\text { Ephemeris } \\
\text { Near Candidate 3 }\end{array}$ \\
\hline MJD range & $52745.790-53140.604$ & $53229.271-53456.688$ & $53562.209-53785.652$ \\
TOAs & 75 & 38 & 28 \\
Epoch (MJD) & 52989.8475 & 53366.3150 & 53635.6772 \\
$\nu(\mathrm{Hz})$ & $0.0908928173(6)$ & $0.0908876402(13)$ & $0.090884059(2)$ \\
$\dot{\nu}\left(10^{-13} \mathrm{~Hz} \mathrm{~s}^{-1}\right)$ & $-1.556(3)$ & $-1.492(6)$ & $-1.485(16)$ \\
$\ddot{\nu}\left(10^{-22} \mathrm{~Hz} \mathrm{~s}^{-2}\right)$ & $1.2(4)$ & $4(2)$ & 0 \\
$d^{3} \nu / d t^{3}\left(10^{-28} \mathrm{~Hz} \mathrm{~s}^{-3}\right)$ & $-0.66(16)$ & $-6.6(1.4)$ & $-19(4)$ \\
$d^{4} \nu / d t^{4}\left(10^{-35} \mathrm{~Hz} \mathrm{~s}^{-4}\right)$ & $-1.0(4)$ & $-18(6)$ & $52(12)$ \\
$\mathrm{RMS} \mathrm{residual}\left(\mathrm{phase}^{\mathrm{b}}\right)$ & 0.0146 & 0.0106 & 0.0152 \\
\hline
\end{tabular}

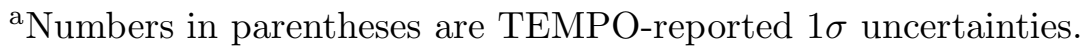

${ }^{\mathrm{b}}$ Entries with the value ' 0 ' are consistent with being zero. 
Table 7. Long-Term Spin Parameters for 1E 1841-045

\begin{tabular}{|c|c|c|c|c|c|}
\hline Parameter & $\begin{array}{c}\text { Ephemeris A } \\
\text { Spanning MJD } \\
51225-52438\end{array}$ & $\begin{array}{c}\text { Ephemeris B } \\
\text { Spanning MJD } \\
52460-52981\end{array}$ & $\begin{array}{c}\text { Ephemeris B2 } \\
\text { Spanning MJD } \\
52610-52981\end{array}$ & $\begin{array}{c}\text { Ephemeris C } \\
\text { Spanning MJD } \\
53030-53816\end{array}$ & $\begin{array}{c}\text { Ephemeris D } \\
\text { Spanning MJD } \\
53829-53983\end{array}$ \\
\hline MJD start & 51224.538 & 52460.000 & 52610.313 & 53030.093 & 53828.808 \\
\hline MJD end & 52437.712 & 52981.186 & 52981.186 & 53815.842 & 53983.431 \\
\hline TOAs & 53 & 19 & 17 & 54 & 11 \\
\hline$\nu(\mathrm{Hz})$ & $0.0849253002(9)$ & $0.084904428(7)$ & $0.084889922(3)$ & $0.084890135(6)$ & $0.084868767(7)$ \\
\hline$\dot{\nu}\left(10^{-13} \mathrm{~Hz} \mathrm{~s}^{-1}\right)$ & $-2.9940(10)$ & $-3.176(2)$ & $-3.179(2)$ & $-3.354(7)$ & $-2.833(9)$ \\
\hline$\ddot{\nu}\left(10^{-22} \mathrm{~Hz} \mathrm{~s}^{-2}\right)$ & $3.30(14)$ & - & - & $16.4(4)$ & - \\
\hline$d^{3} \nu / d t^{3}\left(10^{-29} \mathrm{~Hz} \mathrm{~s}^{-3}\right)$ & $0.9(2)$ & - & - & $-2.81(11)$ & - \\
\hline$d^{4} \nu / d t^{4}\left(10^{-36} \mathrm{~Hz} \mathrm{~s}^{-4}\right)$ & $-2.3(2)$ & - & - & - & - \\
\hline$d^{5} \nu / d t^{5}\left(10^{-43} \mathrm{~Hz} \mathrm{~s}^{-5}\right)$ & $1.5(3)$ & - & - & - & - \\
\hline$d^{6} \nu / d t^{6}\left(10^{-51} \mathrm{~Hz} \mathrm{~s}^{-6}\right)$ & $-3(2)$ & - & - & - & - \\
\hline$\Delta \nu_{d}^{\mathrm{b}}(\mathrm{Hz})$ & - & $8.1(6) \times 10^{-7}$ & - & - & - \\
\hline$t_{d}^{\mathrm{b}}$ (days) & - & $43(3)$ & - & - & - \\
\hline Epoch (MJD) & 51618.000 & 52464.00448 & 52997.0492 & 52997.0492 & 53823.9694 \\
\hline RMS residual (phase) & 0.029 & 0.025 & 0.028 & 0.033 & 0.022 \\
\hline
\end{tabular}

${ }^{\mathrm{a}}$ Numbers in parentheses are TEMPO-reported $1 \sigma$ uncertainties.

${ }^{\mathrm{b}}$ Parameters held fixed at values determined from local glitch fits as shown in Table 8 
Table 8. Local Ephemerides of 1E 1841-045 Near Glitch Epochs ${ }^{\mathrm{a}}$

\begin{tabular}{lccc}
\hline \hline \multicolumn{1}{c}{ Parameter } & Ephemeris & Ephemeris & Ephemeris \\
& Near Glitch 1 & Near Glitch 2 & Near Glitch 3 \\
\hline MJD range & $52001.684-52981.186$ & $52773.845-53244.179$ & $53579.360-53970.681$ \\
TOAs & 30 & 26 & 31 \\
Epoch (MJD) & 52464.00448 & 52997.0492 & 53823.9694 \\
$\nu(\mathrm{Hz})$ & $0.084903950(2)$ & $0.084889815(3)$ & $0.084868657(4)$ \\
$\dot{\nu}\left(10^{-13} \mathrm{~Hz} \mathrm{~s}^{-1}\right)$ & $-2.8980(10)$ & $-3.162(3)$ & $-2.872(4)$ \\
Glitch Epoch $(\mathrm{MJD})$ & 52464.00448 & 52997.0492 & 53823.9694 \\
$\Delta \nu(\mathrm{Hz})$ & $4.78(7) \times 10^{-7}$ & $2.08(4) \times 10^{-7}$ & $1.18(7) \times 10^{-7}$ \\
$\Delta \dot{\nu}\left(\mathrm{Hz} \mathrm{s}{ }^{-1}\right)$ & $-2.78(2) \times 10^{-14}$ & $4(3) \times 10^{-16}$ & $2(1) \times 10^{-15}$ \\
$\Delta \nu_{d}(\mathrm{~Hz})$ & $8.1(6) \times 10^{-7}$ & - & - \\
$t_{d}($ days $)$ & $43(3)$ & - & - \\
RMS residual (phase) & 0.022 & 0.015 & 0.022 \\
\hline
\end{tabular}

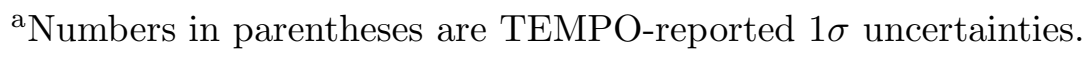

